\title{
SIA: Selection Inference Using the Ancestral Recombination Graph
}

\author{
Hussein A. Hejase ${ }^{1^{*}}$, Ziyi Mo $^{1,2^{\star}}$, Leonardo Campagna ${ }^{3,4}$, Adam Siepe ${ }^{1}$
}

\author{
${ }^{1}$ Simons Center for Quantitative Biology, Cold Spring Harbor Laboratory, Cold Spring Harbor, NY, USA \\ ${ }^{2}$ School of Biological Sciences, Cold Spring Harbor Laboratory, Cold Spring Harbor, NY, USA \\ ${ }^{3}$ Fuller Evolutionary Biology Program, Cornell Lab of Ornithology, Ithaca, NY, USA \\ ${ }^{4}$ Department of Ecology and Evolutionary Biology, Cornell University, Ithaca, NY, USA \\ *These authors contributed equally \\ Corresponding author: Adam Siepel (asiepel@cshl.edu)
}

\section{Keywords}

ancestral recombination graph, machine learning, positive selection, selective sweep

\section{Abstract}

2 Detecting signals of selection from genomic data is a central problem in population genetics.

3 Coupling the rich information in the ancestral recombination graph (ARG) with a powerful and

4 scalable deep learning framework, we developed a novel method to detect and quantify positive selection: Selection Inference using the Ancestral recombination graph (SIA). Built on a Long Short-Term Memory (LSTM) architecture, a particular type of a Recurrent Neural Network (RNN), SIA can be trained to explicitly infer a full range of selection coefficients, as well as the allele frequency trajectory and time of selection onset. We benchmarked SIA extensively on simulations under a European human demographic model, and found that it performs as well or better as some of the best available methods, including state-of-the-art machine-learning and ARG-based

11 methods. In addition, we used SIA to estimate selection coefficients at several loci associated

12 with human phenotypes of interest. SIA detected novel signals of selection particular to the

13 European (CEU) population at the MC1R and $A B C C 11$ loci. In addition, it recapitulated signals of 14 selection at the LCT locus and several pigmentation-related genes. Finally, we reanalyzed 15 polymorphism data of a collection of recently radiated southern capuchino seedeater taxa in the 16 genus Sporophila to quantify the strength of selection and improved the power of our previous 17 methods to detect partial soft sweeps. Overall, SIA uses deep learning to leverage the ARG and 18 thereby provides new insight into how selective sweeps shape genomic diversity. 


\section{Introduction}

21 The ability to accurately detect and quantify the influence of selection from genomic sequence

22 data enables a wide variety of insights, ranging from understanding historical evolutionary events

23 to characterizing the functional and disease relevance of observed or potential genetic variants.

24 Adaptive evolution is driven by increases in frequency of alleles that enhance reproductive fitness.

25 In addition, alleles experiencing such positive selection often provide insights into the functional

26 or mechanistic basis of phenotypes of interest. Examples of genetic determinants of important

27 phenotypic traits under selection in human populations include a family of mutations in the

28 hemoglobin- $\beta$ cluster, which confer resistance to malaria and are at high frequencies in many

29 populations [1,2], loci controlling growth factor signaling pathways that contribute to short stature

30 in Western Central African hunter-gatherer populations [3,4], as well as mutations in several

31 genes involved in immunity, hair follicle development, and skin pigmentation [5] (reviewed in refs.

$32[6-9])$.

34 Population genetic methods predominantly identify positive selection through the detection of selective sweeps. As the frequency of an advantageous allele increases, linked variants in the vicinity can "hitchhike" to high frequency, leading to local reductions in genetic diversity. Previous approaches to detecting selective sweeps (such as traditional summary statistics [10], approximate likelihood and Approximate Bayesian Computation (ABC) methods [11], or

39 supervised machine learning $(M L)$ methods $[12,13])$ exploit the effect of genetic hitchhiking on the 40 spatial haplotype structure and site frequency spectrum (SFS). Summary statistics have the

41 advantage of being fast and easy to compute, but may confound the effects of selection on genetic 42 diversity with the effects of complex demographic histories including bottlenecks, population 43 expansions and structured populations. Besides, they cannot easily be used to estimate the value 44 of the selection coefficient. Approximate likelihood and ABC methods, on the other hand, can 
45 provide an estimate of the strength of selection by aggregating multiple summary statistics [11],

46 but can be prohibitively computationally expensive when applied at a large scale. ML methods for

47 inferring selection can be more scalable, and can capture complex nonlinear relationships among

48 features. With the exception of a handful of recently developed methods that operate on the

49 multiple sequence alignment itself [14,15], however, the majority of $M L$ approaches to selection

50 inference solely make use of traditional summary statistics as features for prediction. In short,

51 previous methods (including $\mathrm{ABC}$ and most ML methods) predominantly rely on low-dimensional

52 summary statistics, which, even in combination, capture only a small portion of the information in

53 the sequence data.

55 Recently, a new generation of inference methods have made it possible to go beyond summary

56 statistics and estimate or sample a full ancestral recombination graph (ARG) [16-18] for a

57 collection of sequences of interest. The ARG is a complex data structure that summarizes the

58 shared evolutionary history and recombination events that have occurred in a collection of DNA

59 sequences, and therefore contains highly informative features that can potentially be leveraged

60 to make accurate inferences about selection. The ARG representation is interchangeable with a

61 sequence of local genealogies along the genome and the recombination events that transform

62 each genealogy to the next. The influence of selection on each allele can be characterized from

63 the ARG, based on departures from the patterns of coalescence and recombination expected

64 under neutrality as reflected in the local genealogies. Traditional ARG inference methods [19-23]

65 were restricted in accuracy and scalability, limiting the practical application of ARGs. Recent

66 advances [24], however, have enabled scalable yet statistically rigorous genome-wide ARG

67 inference with dozens of genomes. Moreover, methods such as Relate [25] and tsinfer [26] have

68 further dramatically improved the scalability of ARG inference to accommodate thousands or even

69 hundreds of thousands of genomes. The latest progress in genealogical inference has paved the

70 way for ARG-based methods to address many different questions in population genetics [24-27]. 
72 One natural way to exploit the richness of the ARG representation in inference of selection would

73 be to extract features from inferred ARGs and feed them into a modern supervised machine-

74 learning framework. Deep-learning methods, in particular, have recently achieved unprecedented

75 success on a variety of challenging problems, including image recognition, machine translation,

76 and game-play [28]. Deep learning is also highly flexible, providing many opportunities for the

77 design of novel model architectures motivated by biological knowledge. An ARG-guided deep-

78 learning model could potentially provide new insight into how natural selection impacts the human

79 genome, human diseases and other phenotypes, and human evolution.

81 With these goals in mind, we developed a new method, called SIA (Selection Inference using the

82 Ancestral recombination graph), that uses a Recurrent Neural Network (RNN) $[29,30]$ to infer the

83 selection coefficient and allele frequency trajectory of a variant that maps to a gene tree

84 embedded in an ARG. Rather than relying on traditional sequence-based summary statistics, SIA

85 makes use of features based on the local genealogies extracted from the ARG. Based on these

86 local topological features, SIA learns to infer the selection coefficient and allele frequency

87 trajectory of a beneficial variant (see Figure 1). As described below, SIA performs well on

88 benchmarks and is reasonably robust to model misspecification. Applying SIA to data from the

891000 Genomes Northern and Western European (CEU) population, we identified new and known

90 loci under positive selection that are associated with a variety of phenotypes and estimated

91 selection coefficients at these loci. In addition, using SIA, we built on our previous work [31] on a

92 bird species-complex in the genus Sporophila by elucidating the strength and targets of selection

93 at specific loci tied to a collection of rapid speciation events. Overall, SIA is the first method that

94 couples ARG-based features with a machine-learning approach for population genetic inference. 
96 Results

97 Methodological overview. SIA is based on an RNN that is trained to predict selection at a genomic

98 site from genealogical features at that site of interest and nearby sites (see Methods for detailed

99 descriptions, see Figure 1 for a conceptual overview of SIA, and Figure S1 for an illustration of

100 ARG features and the RNN architecture). Based on the demography of a particular population of

101 interest, training data including genomic regions under various strengths of selection are

102 simulated. The ARG is then inferred from each simulated data set. ARG-level statistics are

103 extracted at the site under selection (or a neutral site) as features to be used as input to the deep-

104 learning model. Specifically, we use lineage counts at a set of discrete time points as a fixed-

105 dimension encoding of a genealogy. The encoding of the genealogy at the focal site as well as

106 similar encodings of flanking genealogies constitute the feature vector for that site. SIA uses a

107 Long Short-Term Memory (LSTM) architecture, designed specifically to handle the temporal

108 nature of the feature set. The LSTM unrolls temporally such that the lineage counts at each time

109 point are fed to the network iteratively. Finally, the model trained on simulations is applied to

110 ARGs inferred from empirical data to identify sweeps, infer selection coefficients, and allele-

111 frequency trajectories.

113 Classification of sweeps. We first compared SIA with several existing methods, including the

114 Tajima's D [10] and H1 [32] summary statistics, iHS [33], a genealogy-based statistic [25] and a

115 summary-statistic-based machine-learning method [12,13] (see Methods), in the classification

116 task of distinguishing hard sweeps from neutrally evolving regions. Our performance comparison

117 was conducted across 16 combinations of selection coefficients and segregating allele

118 frequencies such that the beneficial site was subjected to selection ranging from weak to strong,

119 resulting in low to high derived allele frequencies (DAFs). Since a priori we expected sweep sites

120 with lower selection coefficients and lower DAFs to be harder to detect, we performed a stratified 
121 analysis of SIA's performance by selection coefficient and DAF. Figure 2 reports the Receiver

122 Operating Characteristic (ROC) curves using simulations based on the CEU demographic model

123 [34] where inferred genealogies were used as input to SIA to account for gene tree uncertainty.

124 As expected, all methods tended to perform better in a regime with higher selection coefficients

125 and DAFs, as indicated by increasing values of the area under the ROC curve (AUROC) statistic

126 from left to right (increasing selection) and from top to bottom (increasing DAF). SIA outperformed

127 the other methods across model conditions, with a more pronounced performance advantage for

128 sites under weaker selection and segregating at lower DAFs (Figure 2). For each given selection

129 coefficient, the AUROC of the Relate tree statistic (shown in red in Figure 2), which measures

130 how unlikely it is that the observed expansion of the derived lineages is purely due to genetic drift,

131 did not substantially improve as the DAF increased. Alleles at higher frequency tend to be older

132 and subjected to drift over longer periods, which may lead to reduced power for Relate to

133 distinguish lineage expansion under selection from the neutral expectation. Consequently, while

134 the ARG-based methods SIA and Relate both outperformed other methods at low DAFs, SIA was

135 alone in maintaining this advantage at higher DAFs.

137 In addition, we validated the ability of SIA to classify genomic regions with additional test sets 138 simulated under a demographic model for southern capuchinos, a group of songbirds in which we 139 previously identified and characterized many examples of sweeps [31], finding a predominance 140 of "soft" rather than "hard" sweeps (meaning that they tend to be based on standing genetic

141 variation rather than new mutations; see Methods). Figure S2 reports the ROC curves for the 142 task of distinguishing partial soft sweeps from neutral regions. Despite soft sweeps being harder 143 to detect, the classifier achieved good performance in the moderate-to-strong selection regimes

$144(s=0.005$ and $s=0.0075)$ where the accuracy ranged between $82 \%$ and $96 \%$, a substantial 145 improvement over the previous accuracy of 56\% [31]. SIA performed particularly well in identifying 146 partial soft sweeps when the site under selection was at a high segregating frequency. For 
example, at segregating frequencies of 0.75 and 0.9 , the performance of SIA ranged between $80 \%$ and $96 \%$ across a variety of selection regimes ( $s=0.0025,0.005$, and 0.0075$)$. The performance of SIA degraded somewhat for weak selection $(s=0.001)$ with an accuracy ranging between $63 \%$ and $74 \%$.

Selection coefficient inference using true gene trees. We assessed the performance of SIA in correctly predicting the selection coefficient and compared it to CLUES [35]. Like SIA, CLUES uses local genealogies based on the ARG to infer a selection coefficient. However, CLUES calculates the likelihood of the genealogy analytically using a hidden Markov model (HMM), and does not rely on simulated training data. In addition, CLUES uses a single genealogy at the focal site, whereas SIA additionally considers flanking trees.

We began by supplying both methods with true genealogies, in order to later disentangle the error deriving from the ARG inference step from other sources of error (see Discussion). We found that SIA identified regions under neutrality with approximately no bias (median inferred $s=7.5 e-$ coefficient (median inferred $s=0.00037$ ) for the weak selection regime (true $s=0.001$ ), likely owing to limits in the training set within that selection regime (see Discussion). We further binned

167 the results by segregating frequency and selection coefficient and found that, in general, the

168 variance in estimates of $s$ for SIA (as well as CLUES) tended to decrease as the segregating 169 frequency of the beneficial allele increased (Figure S3).

171 CLUES performed roughly similarly to SIA in this experiment, but tended to slightly overestimate $172 s$ for the neutral regions (i.e., true $s=0$ ) and underestimate $s$ for the moderate to high selection 
173 regimes (i.e., true $s=0.005,0.0075$, and 0.01). Under these conditions, SIA's median predictions

174 of $s$ were noticeably closer to the true values (Figure 3A). At the same time, CLUES performed 175 slightly better than SIA in weak selection regimes (i.e., true $s=0.001$ and 0.0025 ) (Figure 3).

176 Overall, SIA (RMSE $=9.52 \mathrm{e}-4)$ achieved a lower error in estimating $s$ than CLUES $(\mathrm{RMSE}=$

$177 \quad$ 1.44e-3), when true genealogies were used as input to both methods (Wilcoxon signed-rank test

178 for difference in mean of squared error, $p=1.25 \mathrm{e}-42)$. This finding potentially reflects the benefit

179 of linkage information utilized by SIA through the additional flanking genealogies (see

180 Discussion).

181

182 Selection coefficient inference using inferred gene trees. To account for gene-tree uncertainty, we next used ARGs inferred with Relate, which is scalable to the size of the training dataset for SIA (see Methods), as input to SIA and CLUES and compared their performance on CEU simulations. Furthermore, we compared both methods to a supervised machine learning method, classification and regression.

Overall, we found that SIA and ImaGene outperformed CLUES in these experiments (Figure 4). CLUES tended to underestimate selection coefficients for the moderate-to-strong selection regimes, to a greater extent compared to the case where true genealogies were used for inference

194 (Figures 3A \& 4A). This decrease in performance of CLUES evidently derives from error at the 195 ARG reconstruction step. SIA, on the other hand, appeared to be more robust to the same ARG 196 reconstruction error. ImaGene performed remarkably similarly to SIA, given that it relies solely on 197 the sequence alignment. SIA exhibited lower error at neutral sites and sites with low-to-moderate 198 values of $s$, whereas ImaGene prevailed at sites under strong selection (Figure 4B). 
199 Nevertheless, SIA showed a slightly smaller overall RMSE (2.75e-3) compared to ImaGene

200 (2.91e-3) (Wilcoxon signed-rank test, $p=6.18 \mathrm{e}-38)$, and in particular, SIA produces estimates of

201 s much closer to 0 for neutral loci. Notably, in this case both SIA and ImaGene were trained with

202 simulations under the same uniform distribution of $s$ values (see Methods). A different choice of

203 training distribution could impact their performance across selection regimes (see Discussion).

204 Furthermore, we binned the results of these methods by both the segregating frequency and the

205 selection coefficient (see Figure S4) and again found that in general they exhibit higher variance

206 under low segregating frequency of the beneficial allele. As before, we also tested our regression

207 framework on true and inferred gene trees of test sets simulated under the S. hypoxantha

208 demographic model (see Figure S5). We found that SIA was approximately unbiased for the

209 moderate $(s=0.005)$ and high $(s=0.01)$ selection regimes but appeared to overestimate the

210 selection coefficient for regions under weak selection ( $s=0.001$ and 0.0025$)$, when both true and

211 inferred genealogies were used as input. Furthermore, SIA appeared to overestimate the

212 selection coefficient for neutral regions when inferred gene trees were used as input, whereas it

213 was approximately unbiased for true gene trees.

215 Performance on selection coefficient prediction with different sample sizes. To explore the

216 tradeoffs associated with the use of larger data sets, we examined the performance of SIA under

217 different sample sizes, assuming a constant-sized demographic model $\left(N_{\mathrm{e}}=10,000\right)$. Figure S6

218 shows the error in selection coefficient inference on a held-out test set, stratified by the age of the

219 allele (panels $\mathbf{A} \& \mathbf{B}$ ) and present-day derived allele frequency (panels C\&D) at the site of interest.

220 We observed that sites with low frequency (AF $<0.33$ ) and more recent (onset $<0.2 \times 2 N_{e}$

221 generations) alleles experience the most significant reduction in error as sample size increases.

222 Notably, the performance of SIA on more ancient alleles (onset $>0.2 \times 2 N_{\mathrm{e}}$ generations) had little

223 to no improvement as the sample size increased from 32 to 254 . These observations are in line

224 with the expectation that having more samples improves the chance of capturing low-frequency 
225 alleles, but provides limited information about more ancient events. The reason for this age-

226 dependency is that, looking backwards in time, most lineages coalesce rapidly and only a few

227 survive to more ancient epochs, in a manner that depends only weakly on the sample size. It may

228 be useful to consider these observations when choosing the sample size for use in studying

229 selection in a particular context (see Discussion).

Inference of allele frequency trajectory. We further adapted the deep-learning architecture of SIA

232 to model the allele frequency (AF) trajectory at a site by retaining the output of the LSTM at each

233 time point (Figure S1, see Methods). We then evaluated the performance of SIA in the inference

234 of the AF trajectory using simulations under the CEU demography across a range of selection coefficients and current DAFs. SIA was largely able to capture the expected trend of more rapidly increasing AF under stronger selection (Figure S7 and S9). In addition, AF estimates by SIA using both true and inferred genealogies were generally unbiased, although AF at more recent time points tended to be slightly underestimated when data was simulated under weaker selection. AF estimates also appeared to be more accurate in terms of variance for alleles under stronger selection (Figure S8 and S10). As expected, the variance of AF estimates tended to

241 increase going further back in time (Figure S8 and S10).

Model performance on simulations with misspecified demographic models. To evaluate the

244 robustness of SIA to mismatches between the demographic parameters used for simulating

245 training data and the true underlying demography of real data, we tested the method on the 246 selection-coefficient inference task with datasets simulated under a range of alternative 247 parameters. Each aspect of this model misspecification was assessed independently of the 248 others. In particular, the misspecified datasets contained simulations under (i) combinations of 249 population mutation $(\theta)$ and recombination $(\rho)$ rates sampled beyond the range used for the 250 training data (Figures S11 and S14), (ii) various alternative demographic scenarios (Figures S12, 
251 S15, and S17), and (iii) various effective population sizes (Figures S13 and S16). We compared

252 the performance of SIA on these misspecified datasets to that of CLUES [35], supplying both

253 methods with the true genealogies. We consider CLUES the "silver standard" when it comes to

254 robustness because it is unsupervised and therefore should not be susceptible to misspecified

255 training data compared to supervised learning methods such as SIA. Overall, we found that both

256 CLUES and SIA were reasonably robust to model misspecification (Figures S11-13), although

257 the performance of both methods inevitably declined when tested on severely misspecified data

258 (Figure S13). Interestingly, SIA tended to overestimate selection coefficient when the true $N_{\mathrm{e}}$ was

259 much smaller than that used for training, and underestimate it when the true $N_{\mathrm{e}}$ was much larger,

260 whereas CLUES did the opposite (Figure S13). Since the CLUES likelihood model of allele

261 frequency transition is parameterized by the population-scaled selection coefficient $(\alpha=2 N s)$, a

262 larger $N_{\mathrm{e}}$ likely appears to CLUES as equivalent to a higher $s$. On the other hand, features used

263 by SIA capture broad information of coalescence and linkage in the ARG, and therefore can be

264 distorted by misspecified $N_{\mathrm{e}}$ in more subtle ways (see Discussion). Using the same misspecified

265 dataset, we also ran SIA with Relate-inferred genealogies and compared its performance to that

266 of the genotyped-based deep-learning model ImaGene [14,15]. In general, SIA appeared to be

267 more robust to model misspecifications, achieving an overall RMSE of $0.00362,0.00318$ and

2680.00374 in the misspecified $\theta / \rho$, demography, and $N_{\mathrm{e}}$ experiments, respectively, compared to

269 ImaGene, whose RMSE was $0.00416,0.00330$ and 0.00462 in the corresponding experiments

270 (Figures S14-16). The advantage of SIA was particularly noticeable in cases of misspecified

271 demographic parameters (Figures S15 \& S16). Notably, SIA exhibited reduced bias when

272 working with inferred genealogies compared to true genealogies, under conditions of extremely

273 mismatched $N_{\mathrm{e}}$ (compare Figures S13 \& S16).

275 Model prediction at genomic loci of interest in CEU population. We then applied the SIA model to 276 identify selective sweeps and infer selection coefficients at selected genomic loci in the 1000 
277 Genomes CEU population. These loci included the canonical example of selection at the MCM6

278 gene, which regulates the neighboring LCT gene and contributes to the lactase persistence trait

279 [36], the $A B C C 11$ gene regulating earwax production, several pigmentation-related genes, as well

280 as genes associated with obesity, diabetes and addiction (Table 1).

For LCT, SIA detected a strong signal of selection at the nearby SNP that has been associated with the lactase persistence trait (rs4988235). At this SNP, SIA inferred a sweep probability close

to 1 and a selection coefficient greater than 0.01 , making this one of the strongest signals of selection in the human genome. A close examination of the local genealogy at this site reveals a clear pattern indicative of a selective sweep - a burst of recent coalescence among the derived lineages (orange taxa are the lineages carrying the derived allele) is clearly visible from the tree

(Figure 5).

At a number of pigmentation genes [37-41], SIA detected signals of moderate selection, including $\operatorname{MC1R}(\mathrm{rs1805007,} \mathrm{P}(\mathrm{sweep})=0.95, \mathrm{~s} \approx 0.0037), K I T L G(\mathrm{rs12821256}, \mathrm{P}(\mathrm{sweep})=0.87, \mathrm{~s} \approx$ 0.0019), ASIP (rs619865, P(sweep)= 0.78, s $\approx 0.0019)$, OCA2 (rs12913832, $\mathrm{P}($ sweep $)=0.75, \mathrm{~s}$ $\approx 0.0056)$ and TYR (rs1393350, $P($ sweep $)=0.62, s \approx 0.0011)$. In addition, SIA identified a weak

294 signal of selection at a SNP in the ABCC11 gene (rs17822931), which influences earwax and 295 sweat production [42], with a selection coefficient of around 0.00035. There are few other 296 estimates for these genes available for comparison, but, notably, our estimate for LCT of $s \approx 0.01$ 297 is consistent with a previous estimate on the order of 0.01-0.1 [36], and with recent studies of 298 ancient DNA samples [43,44] suggesting a value closer to 0.01 . Our estimates suggest that 299 selection at the pigmentation loci is considerably weaker than at $L C T$, in contrast to previous 300 estimates for these loci, which covered a wide range but were generally considerably larger 301 (ranging from 0.02-0.1) [45]. Interestingly, CLUES estimated $s$ at the OCA2 locus to be on the 302 order of 0.001 (roughly similar to SIA's estimate of 0.0056 ), but $s$ at the KITLG, ASIP, TYR loci to 
be greater than 0.01 (in comparison to SIA's considerably smaller estimates of $0.0019,0.0019$, and 0.0011) [35]. The apparent discrepancy between the estimates may be partially due to the fact that the two methods used samples from two different populations (CEU for SIA and GBR/British for CLUES).

On the other hand, SIA did not detect significant evidence of positive selection at several diseaseassociated loci (rs7903146/TCF7L2, rs1800497/ANKK1, and rs9939609/FTO) or at several other pigmentation loci (rs13289810/TYRP1, rs1003719/TTC3, and rs7495174/OCA2) (Table 1).

311 Notably, allele frequencies at these six loci tend to be similar in African and European populations 312 [46], suggesting that they are not likely to be under strong environment-dependent positive selection, although it is possible that they have experienced very recent selective pressure that

314 SIA lacks the power to detect (see Discussion). Notably, TYRP1 and TTC3 also lacked signals of selection in the CLUES analysis. Compared to the genealogies at sweep sites (Figure 5), the trees at these putatively neutral loci lack the distinctive signature of recent bursts of coalescence

317 among derived lineages (Figure 6).

Southern capuchino species analysis. Our previous study of southern capuchino seedeaters made use of the full ARG and machine learning to detect and characterize selective sweeps, and

321 suggested that soft sweeps are the dominant mode of adaptation in these species (see Methods 322 for more details). To further characterize the targets and strengths of positive selection in these 323 species, we applied SIA to polymorphism data [47] for S. hypoxantha, and adopted a conservative 324 approach by reporting only sites with DAF $\geq 0.5$, SIA-inferred $s \geq 0.0025$, and SIA-inferred sweep 325 probability $\geq 0.99$ (see Methods). In addition to loci near top $F_{\text {ST }}$ peaks and known pigmentation326 related genes (Table 2), we identified many more sites under positive selection located outside 327 the previously scanned $F_{\text {ST }}$ peaks, amounting to a total of 15,551 putative partial soft sweep sites 328 across the 333 scanned scaffolds for S. hypoxantha. These sites can be prioritized for further 
evaluation and downstream analysis. Notably, SIA enabled us to distinguish between selection at regulatory and coding sequences, and we found that sweep loci near $F_{\mathrm{ST}}$ peaks and pigmentation

331 genes fall mostly in non-coding regions (Table 2). We additionally surveyed all putative sweep

332 sites identified by SIA and found that they are indeed enriched in non-coding regions (Fisher's 333 exact test, $p=6.80 \times 10^{-5}$ ), particularly noticeable in the "near-coding" regions (Figure S21).

334 Consistent with the observation that the most highly differentiated SNPs among taxa are non335 coding $[47,48]$ our finding suggests that positive selection may act on cis-regulatory regions to 336 drive differentiation and the subsequent speciation process. Furthermore, we examined many 337 individual predictions in detail, considering the local trees inferred by Relate at these highconfidence predictions (Figure 7). We found, in numerous cases, that these sweeps had distinct genealogical features, displaying evidence of a burst of coalescence events, corresponding to unusually large and young clades. Prominent examples include predictions near pigmentationrelated genes ASIP, KITL, SLC45A2, and TYRP1.

\section{Discussion}

344 The ARG is useful for addressing a wide variety of biological questions ranging from inferring

345 demographic parameters to estimating allele ages. SIA exploits the particular utility of the ARG

346 for accurate inference of positive selection in a way that makes use of the full dataset, as opposed

347 to traditional summary statistics, which necessarily discard substantial information. Direct use of

348 the ARG improves upon traditional summary statistics in two key ways. First, it enables

349 consideration of the temporal distribution of coalescence and recombination events in the history

350 of the analyzed sequences, in contrast to traditional summary statistics that simply average over

351 these coalescence and/or recombination events. In addition, ARG-based methods provide better 352 spatial resolution by separately examining individual genealogies and the recombination 353 breakpoints between them, rather than averaging across windows containing unknown numbers 
354 of genealogies. These detailed patterns of coalescences and linkage enable the ARG-based

355 approaches to capture a more localized and fine-grained picture of selection (e.g. infer selection

356 coefficient and allele frequency trajectory) as well as to achieve a better classification

357 performance. This performance advantage is particularly noticeable at lower DAFs and when

358 selection is weak, a regime where previous methods for selection inference fall short (Figure 2).

At the same time, the supervised machine-learning approach sets SIA apart from another ARGbased method, CLUES, which approximates a full likelihood function for ARGs in the presence of selection using importance sampling and a HMM. Although the accuracy of both SIA and CLUES degraded when using inferred genealogies compared to true genealogies, reflecting the error and uncertainty at the ARG inference step, SIA appeared to be more robust to gene tree uncertainty

(Figures 3 and 4). One possible reason for this observation is that CLUES effectively assumes that the selection coefficient at the focal site is conditionally independent of the flanking trees given the focal tree. This assumption should hold in the presence of fully specified genealogies, but it may make CLUES more sensitive to errors in the inferred genealogies. In other words, through its use of supervised learning, SIA may be able to compensate for the effects of genealogy inference error on its estimation of the selection coefficient by also directly considering

371 the flanking trees and LD-related patterns among them. Still, the drop in accuracy observed

372 across methods underscores the dependency of ARG-based approaches on the ARG inference 373 method. For this reason, we anticipate that SIA may benefit substantially from further 374 improvement in ARG inference tools (see ref. [9]).

376 The ARG-based feature set distinguishes SIA from other supervised machine learning 377 approaches for characterizing selective sweeps. SIA uses local topological features of the ARG, 378 which are more informative than the SFS- or LD-based summary statistics employed by machine 379 learning methods such as S/HIC, SFselect, and evolBoosting. Using simulations, we 
demonstrated that the SIA classifier outperformed a deep-learning method that aggregates these

381 traditional summary statistics (Figure 2). We also compared SIA with ImaGene, which represents

382 another flavor of supervised learning methods, inspired by the recent rise of CNNs for image

383 recognition. ImaGene encodes sequence alignments as images for powerful population genetic

384 inferences with CNNs and provides a state-of-the-art benchmark to compare against. We found

385 that ImaGene performs remarkably well across a wide range of simulations, but SIA does appear

386 to be somewhat less biased and more robust to model misspecification than ImaGene. The

387 evolutionary information in the ARG is implicit in the sequence alignment but some of this

388 information may be difficult for a brute-force machine learning model to discover directly.

390 We demonstrated that utilizing the ARG granted SIA considerably improved performance over 391 deep learning models solely employing traditional summary statistics. However, a possible 392 drawback of an ARG-based model is the potentially prohibitive computational overhead incurred by ARG inference, especially as sample size grows. Picking a sample size when running SIA

394 involves a tradeoff between scalability (fewer samples, faster ARG inference) and performance 395 (more samples, slower ARG inference). We have found that SIA can infer selection coefficients reasonably well with as few as 16 haplotypes. Including more samples did improve performance

397 but with a sublinear reduction in error (Figure S6). Therefore, a sample size from a few dozen to 398 a few hundreds - well within the capabilities of most modern ARG inference methods — strikes 399 a good balance between performance and scalability. Moreover, we found that larger sample 400 sizes improved prediction performance primarily for alleles at lower frequencies but had little 401 impact on the performance for more ancient alleles (as most lineages would have already 402 coalesced going further back in time) (Figure S6). This observation suggests that the choice of 403 the sample size when applying SIA should be guided by the biological question of interest — 404 ancient selection can be studied with just a handful of samples, whereas a larger sample size is 405 better suited to detect more recent sweeps. 
407 Like other supervised learning methods, SIA relies on simulations to generate training data, and

408 therefore could be biased by subjective choices of simulation parameters. For example, SIA and

409 ImaGene cannot make accurate predictions of selection coefficients outside the range 410 represented in the training data (Figure S18), whereas unsupervised methods such as CLUES

411 are not limited to a pre-defined range (Figure S19). This problem could be circumvented by 412 training on an extended range of $s$. Similarly, the tendency of SIA to underestimate the selection 413 coefficient for sites under weak selection (Figures 3,4 ) could be mitigated by augmenting the 414 training set with simulations densely sampled from the weak selection regime. A more subtle 415 issue, however, arises when the underlying generative process of the real data does not match 416 the assumptions made for the simulations of the training data, potentially compromising the 417 accuracy of the method when applied to real data. Thus, we tested SIA on simulations with 418 parameters mismatching those used in the training procedure. In general, we found that SIA was 419 fairly robust to alternative parameter values, although, as expected, performance did degrade 420 somewhat under severely misspecified models. Notably, SIA achieved a similar level of

421 robustness to model parameter misspecification as the unsupervised (i.e. not relying on training 422 data) likelihood method CLUES, yet outperformed the supervised deep learning method 423 ImaGene.

425 Applying SIA to the CEU panel from the 1000 Genomes Project yielded several noteworthy 426 findings at loci with known ties to phenotypes of interest. In addition to confirming the canonical 427 signal of selective sweep at the LCT locus, SIA detected a novel signal of selection at a GWAS 428 SNP in the MC1R gene associated with red hair color, contrasting a previous study that could not 429 find evidence of selection at MC1R in the European population [49]. The derived allele at this 430 locus segregates at around $10 \%$ in the CEU population but is nearly absent in non-European 431 populations [46]. In addition, at the MC1R locus the Relate test statistic for selection [25], which 
432 tends to perform particularly well at low segregating frequencies (Figure 2), falls slightly below

433 the significance threshold of 0.05 , supporting the evidence of positive selection at this locus. SIA

434 also detected evidence of selection at a SNP in the $A B C C 11$ gene reported to be the determinant

435 of wet versus dry earwax as well as sweat production, mirroring the signal of selection previously

436 found in the East Asian population [50], although selection in the CEU population appeared to be

437 much weaker. In addition, SIA identified selection at a few other pigmentation-related loci, yet

438 determined previously identified SNPs in the TYRP1 and TTC3 genes to be largely free from

439 selection (Table 1). These results were consistent with a previous study [35], which reported

440 similar results for these pigmentation-related loci, albeit in a slightly different population (GBR).

441 SIA notably did not detect positive selection at GWAS loci in the TCF7L2 gene associated with

442 type-2 diabetes, the ANKK1 gene implicated in addictive behaviors, and the FTO gene associated

443 with obesity. Overall, this empirical study with the 1000 Genomes CEU population has illustrated

444 how SIA can be applied to assess natural selection at the resolution of individual sites, suggesting

445 that it may be useful in prioritizing GWAS variants for further scrutiny.

447 In our previous work on southern capuchino seedeaters [31] (see Methods), we applied newly 448 developed statistical methods for ancestral recombination graph inference and machine-learning 449 for the prediction of selective sweeps. We found evidence suggesting that a substantial fraction 450 of soft sweeps are partial but had limited power to identify them (i.e. average accuracy of 56\%). 451 SIA considerably improved our characterization of positive selection in the southern capuchino 452 species in two key ways. The SIA framework performs inference of selection directly from 453 genealogies instead of traditional summary statistics, and in doing so achieved an accuracy of up 454 to $96 \%$ in detecting partial soft sweeps. Consequently, we found abundant evidence of soft 455 sweeps beyond the previously scanned $F_{\mathrm{ST}}$ peaks, and additionally were able to estimate their 456 selection coefficients. Importantly, SIA also took the analysis of selection beyond broad genomic 457 windows containing sweeps to the identification of specific putative causal variants. We took 
advantage of this substantial improvement in genomic resolution and analyzed the distribution of these sweep sites, which revealed that positive selection on regions that likely contain cis-

460 regulatory elements plays a role in driving the differentiation and speciation of southern capuchino

461 seedeaters.

463 While we believe SIA represents an important step forward in the use of the ARG for machine464 learning-based selection inference, there remain several possible avenues for improvement. For 465 example, SIA currently uses a point-estimate of the ARG, rather than a distribution, and therefore 466 does not explicitly take gene-tree uncertainty into account. We plan to improve SIA by using 467 strategies for inferring approximate posterior distribution of ARGs (e.g., [24]), as well as designing 468 better algorithms for ARG reconstruction that balance accuracy with scalability and can handle 469 thousands of genomes. In addition, the SIA framework was applied in the context of single-locus 470 selective sweeps, but could be extended to study polygenic selection, by making use of summary 471 statistics from genome-wide association studies (as in [51]) and adapting the architecture of our

472 neural network to account for selection acting at multiple sites. Finally, the robustness of SIA to 473 model misspecifications can be further improved by ensuring the simulated data is generated 474 under a distribution that is compatible with the real target data set. We anticipate that the continual 475 advancement in ARG inference methods has the potential to open up many new applications for 476 this flexible and powerful model of ARG-based deep learning in population genetics.

479 Simulated datasets used for training and testing the selective sweep model. Training and testing 480 data sets were generated using discoal [52] by simulating 1,000,000 regions of length $100 \mathrm{~kb}$ for 481 each model we considered (i.e., "neutral" or "hard sweep"). Aside from these regions, 2,000 were 482 simulated for validation and 5,000 were simulated for testing. The number of sampled sequences 
was selected to match the number of individuals in the CEU population in the 1000 Genomes dataset. Thus, a total of 198 haploid sequences were sampled. Simulations used a demographic model based on European demography [34]. In non-neutral simulations, selection was applied to a single focal site located in the middle of the simulated region. We sampled each of the main 487 demographic and selection parameters from a uniform distribution: (1) mutation rate $\mu \sim U(1.25 e-$

$489 U(0.0001,0.02)$, and (4) segregating frequency of the site under selection $f \sim U(0.01,0.99)$.

ARG Feature Extraction. For each target variant, we extracted the corresponding gene tree from

492 the ARG, then overlaid it with 100 discrete timepoints. These timepoints were fixed across all trees in an approximately log-uniform manner that resulted in finer discretization of more recent time scales (as in [24]). We considered biallelic sites only and assumed no recurrent mutations; thus each mutation was assumed to occur on the branch of the tree where the ancestral allele switches to the derived. For each timepoint, we calculated the number of active ancestral and

497 derived lineages. Furthermore, we computed the number of all active lineages (not distinguishing between ancestral and derived) at the same set of predefined timepoints in the two left and right flanking gene trees to account for linkage disequilibrium. Together, these features were summarized in a 600-dimensional feature vector, which was then used as input to an RNN. The

501 feature of a simulated sweep region was extracted from the sweep site (by default at the center 502 in all simulations) whereas the feature of a simulated neutral region was extracted from a variant 503 site (randomly chosen) with a pre-defined matched derived allele frequency. The features for each 504 genomic locus of interest in the CEU population were extracted from all variant sites at that locus 505 having a derived allele frequency of $>0.05$.

507 Training an RNN to predict different modes of selection. An RNN was applied to the simulated 508 training data sets to learn a classification or regression model for the task at hand. We used a 
Long Short-Term Memory (LSTM), a particular form of RNN, to accommodate the temporal nature

510 of our features and account for long-term dependencies and the vanishing gradient problem

511 observed in traditional RNNs. Our model had 100 timepoints with the final target output depending

512 on the use of classification or regression. For the classification task, the final target output is a

513 label for a binary classification problem predicting whether a region is under selection or neutrality.

514 For the regression task, the final target output is a continuous value, representing the selection

515 coefficient or the time of selection onset. We also took a many-to-many approach to model the

516 allele-frequency trajectory for the site under selection. The Keras software was used to train and

517 test the model. We used a two-stacked LSTM to account for greater model complexity where the

518 number of units in each stack was set to 100 and the hyperbolic tangent (tanh) was used as an

519 activation function. The Adam optimization method with its default operating parameters was used

520 to update the network weights. For the classification task, the Softmax activation function was

521 applied on the final dense layer and the binary_crossentropy was used to compute the cross-

522 entropy loss between true labels and predicted labels. For the regression task, the linear

523 activation function was applied on the final dense layer and the mean_squared_error function was

524 used.

525

526 Estimation of Confidence Intervals. To turn our single-valued regression model into one capable

527 of returning a distribution of predictions of $s$, we reused the dropout technique that is typically

528 used during training. Dropout enables a fraction of nodes to be randomly "turned off" in a certain

529 layer, which assists in the regularization of the model and helps prevent overfitting. We applied

530 dropout during inference, enabling us to sample a "thinned" network to generate a sample

531 prediction. By repeatedly sampling thinned networks, we generated a distribution of predictions

532 and then computed confidence intervals based on this distribution [53]. 
534 ARG Inference. Relate [25] (v1.0.17) was used for inferring ARGs underlying simulated genomic

535 samples as well as the CEU population in the 1000 Genomes dataset. For simulations under the

536 Tennessen et al. demography [34], Relate was run with the true simulation parameters $(\mu, \rho$ and

$537 N_{e}$ ) specified; whereas for genomic loci of the CEU population, Relate was run with a mutation

538 rate of $2.5 \times 10^{-8} /$ base/generation (-m $\left.2.5 \mathrm{e}-8\right)$, a constant recombination map of $1.25 \times 10^{-8}$

539 /base/generation and a diploid effective population size of 188,088 (-N 376176). The choice of

540 mutation rate follows [35] based on estimates from [54]. Although some more recent estimates

541 have been lower [55], these differences in mutation rate are unlikely to have a major effect on our

542 selection inference since SIA appears to be fairly robust to misspecification of mutation rate

543 (Figures S11 \& S14). For simulations and genomic loci of the S. hypoxantha population, Relate 544 was run with $\mu=\rho=1 \times 10^{-9} /$ base/generation and a diploid $N_{e}$ of 130,000 . The branch lengths of

545 Relate-inferred genealogies were estimated iteratively with the `EstimatePopulationSize.sh`script

546 in the Relate package. Specifically, population size history was inferred from the ARG, the branch

547 lengths are then updated for the estimated population size history and these steps are repeated

548 until convergence. This was done for a default of 5 iterations (--num_iter 5).

Alternative methods for selection inference. To benchmark the performance of SIA for

551 classification of sites under neutrality versus selective sweep, we ran the following methods:

552 Tajima's D [10], H1 [32], iHS [33], a summary statistics-based deep learning model, and a tree-

553 based statistic that is part of the Relate [25] program. Tajima's D, H1 and iHS were calculated

554 with the scikit-allel package. Haplotypes of the entire $100 \mathrm{~kb}$ simulated genomic segment were

555 used for Tajima's D and H1 calculations. The unstandardized iHS was computed at every site

556 with minor allele frequency $>5 \%$, with respect to all other sites in the genomic segment

557 (min_maf=0.05, include_edges=True). iHS scores of all sites were then standardized in 50 allele-

558 frequency bins. Finally, the iHS score of a genomic region was taken to be the mean of the iHS

559 scores of all of its variant sites. For the summary statistics-based deep learning model, we made 
560 use of the summary statistics used by S/HIC $[12,13]$ as features for our deep learning architecture.

561 These included 11 sequence-based summary statistics (see Figure 3 in [56]) which were used 562 as features for our deep learning model to distinguish among the two classes at hand (selective 563 sweep versus neutral drift). All statistics were collected along five consecutive 20-kb windows with

564 the objective of identifying possible sweeps induced by a positively selected mutation in the third 565 (middle) window. Some of these summary statistics corresponded to standard measures of 566 diversity, such as ss (the number of segregating sites), $\pi$ [57], Tajima's D [10], $\theta_{W}$ [58], $\theta_{H}$ [59], 567 the number of distinct haplotypes [60], $\mathrm{H} 1, \mathrm{H} 12, \mathrm{H} 2 / \mathrm{H} 1$ [32], $\mathrm{Z}_{\mathrm{ns}}$ [61], and maximum value of $\omega$ 568 [62]. For each of these statistics, we computed an average value for each of the five $20 \mathrm{~kb}$ 569 windows for the simulated population. Finally, each summary statistic was normalized by dividing 570 the value recorded for a given window by the sum of values across all five windows. The Relate 571 tree-based selection test was performed with an add-on module (DetectSelection.sh) using the 572 inferred genealogy with calibrated branch lengths at a site of interest, yielding a $\log _{10} p$-value for 573 each site. We also compared the performance of SIA for selection coefficient inference to that of 574 CLUES [35] and a genotype-based convolutional neural network (CNN) framework $[14,15]$. 575 Selection coefficient inference from true genealogies was performed with clues-v0 576 (https://github.com/35ajstern/clues-v0). Transition probability matrices were built on a range of 577 selection coefficients $[0,0.05]$ at increments of 0.0001 and present-day allele frequencies [0.01, 578 0.99] at increments of 0.01. Selection-coefficient inference from Relate inferred genealogies was 579 performed with CLUES (https://github.com/35ajstern/clues). Branch lengths of the genealogy at 580 the site of interest were resampled with Relate for $600 \mathrm{MCMC}$ iterations, and CLUES was run 581 with the following arguments: '--tCutoff 10000 --burnin 100 --thin 5`. For the genotype-based CNN 582 model, each simulated genomic segment was preprocessed by first sorting the haplotypes and 583 then converting the segment to a fixed-size genotype matrix. Haplotype sorting was performed 584 by 1) calculating the pairwise manhattan distances between haplotypes, 2) setting the haplotype 585 with the smallest total distance to all other haplotypes as the first haplotype, and 3) sorting the 
remaining haplotypes in increasing distance to the first haplotype. To convert the sorted

587 haplotypes to a fixed-size genotype matrix, centered on the middle variant of a simulated region,

588 up to 180 variants on each side were retained. Variants beyond 180 were discarded and if there

589 were fewer than 180, the missing variants were padded with zeros. Ancestral and derived alleles

590 were coded with 0's and 1's, respectively. Consequently, each simulated genomic region was

591 encoded as a $(198 \times 360)$ binary matrix, along with a real-valued vector encoding the genomic

592 positions of the variants in the matrix. The CNN model had a branched architecture — one branch

593 with five 1D convolution layers taking the genotype matrix as input and another branch with a fully

594 connected layer taking the vector of variant positions as input. The output of the two branches

595 was flattened, concatenated and fed into 3 fully connected layers, followed by a linear output layer

596 to predict selection coefficient (Figure S20).

598 Evaluation metrics. To evaluate the performance of SIA's classification model and alternative

599 methods, we computed a receiver operating characteristic (ROC) curve for the binary class at

600 hand ("neutral" or "sweep"), to provide a more complete summary of the behavior of different

601 types of errors. We further assessed the performance of SIA and alternative methods in terms

602 of correctly predicting the selection coefficient numerically using mean absolute error (mae),

603 root mean square error (rmse), coefficient of determination $\left(r^{2}\right)$, and visually using a box plot that

604 compares the simulated ground truth against the predictions by the method at hand.

Robustness study. We carried out an extensive analysis of the robustness of our approach,

607 considering not only alternative demographic parameters (such as population size), but also

608 alternative parameters for recombination rate, mutation rate, time of selection onset, and selection

609 coefficients. In all cases, we took care to test our prediction methods under parameters well 610 outside the range used in training.

611 
612 Analysis of CEU population in 1000 Genomes data. We applied SIA to infer selection coefficients

613 and allele frequency trajectories in the 1000 Genomes [63] CEU population at 13 genomic loci

614 with known association to phenotypes, some of which were previously identified as likely targets

615 of positive selection (Table 1). For each gene of interest, the ARG was inferred with Relate from

616 SNPs within a $2 \mathrm{Mb}$ window centered at the gene. Once the ARG was inferred, only SNPs with

617 valid ancestral allele ('AA' INFO field in the vcf file) were retained for downstream analysis.

618 Following the aforementioned protocol (see ARG feature extraction), features at all variant sites

619 in the $2 \mathrm{Mb}$ window above a derived allele frequency threshold of 0.05 were extracted. Lastly, the

620 SIA model was applied to classify neutrality versus selection, and infer selection coefficient and

621 allele frequency trajectory at each site.

Localizing sweeps in southern capuchino seedeaters. We recently applied a combination of ARG

inference and machine-learning methods for identifying selective sweeps to study previously

identified "islands of differentiation" in southern capuchino seedeaters and distinguish among soft sweeps), validation (1000 neutral; 1000 soft sweeps), and testing (2,500 neutral; 2,500 soft sweeps) data sets for SIA under a demographic model inferred by G-PhoCS [64]. Simulations

631 were performed using discoal with the following parameters: (1) mutation rate $\mu=1 e-9$, (2)

632 recombination rate $\rho=1 \mathrm{e}-9$, (3) derived $N_{\mathrm{e}}=130,000$, (4) root divergence time $=1,850,000$

633 generations ago, (5) root $N_{\mathrm{e}}=1,450,000,(6)$ ancestral divergence time $=44,000$ generations ago,

634 (7) ancestral $N_{e}=14,380,000$, (8) selection coefficient $s \sim U(0.001,0.02)$, (9) initial frequency at 635 which selection starts acting on the allele $f_{\text {init }} \sim U(0.01,0.05)$, and (10) segregating frequency of 636 the site under selection $f \sim U(0.25,0.99)$. A total of 56 haploid sequences were sampled from 637 each simulation, matching the number of S. hypoxantha individuals (28) in the real data. The SIA 
638 model for S. hypoxantha was built, trained and evaluated in an otherwise similar fashion to that

639 for the CEU population as outlined above.

640

641 Using a subset of polymorphism data from [47] of 28 S. hypoxantha and 2 S. minuta individuals,

642 we applied our trained model to localize selective sweeps in S. hypoxantha on 19 scaffolds that

643 contain top $F_{\mathrm{ST}}$ peaks in at least one pairwise species comparison [48] and/or harbor known

644 pigmentation-related genes such as ASIP (located on scaffold 252; induces melanocytes to

645 synthesize pheomelanin instead of eumelanin), KITL (located on scaffold 412; stimulates

646 melanocyte proliferation), SLC45A2 (located on scaffold 404; transports substances needed for

647 melanin synthesis), and CAMK2D (located on scaffold 1717; cell communication), as well as 316

648 scaffolds that i) are longer than $100 \mathrm{~kb}$, ii) contain more than 1,000 variants, and iii) where more

649 than $95 \%$ of sites have a consensus ancestral allele, as determined by four identical haplotypes

650 for two individuals from the outgroup species S. minuta. The ARG was inferred with Relate for

651 each scaffold independently. Once the ARG was inferred, the SIA model was applied to sites with

652 consensus ancestral allele for classification and selection coefficient inference.

\section{Acknowledgments}

655 The authors would like to acknowledge Noah Dukler for help with Figure 1 preparation. This 656 research was supported by US National Science Foundation grant (NSFDEB) 1555769, US 657 National Institutes of Health grant R35-GM127070, the CSHL School of Biological Sciences 658 Gladys \& Roland Harriman Fellowship, and the Simons Center for Quantitative Biology at Cold 659 Spring Harbor Laboratory. The content is solely the responsibility of the authors and does not 660 necessarily represent the official views of the US National Institutes of Health or the US National 661 Science Foundation. 


\section{Availability of data and materials}

664 The scripts used for analyses in this study are available at github.com/CshlSiepelLab/arg-

665 selection under a GNU GPLv3 license.

\section{References}

1. Ohashi J, Naka I, Patarapotikul J, Hananantachai H, Brittenham G, Looareesuwan S, et al. Extended Linkage Disequilibrium Surrounding the Hemoglobin E Variant Due to Malarial Selection. Am J Hum Genet. 2004;74: 1198-1208. doi:10.1086/421330

2. Currat M, Trabuchet G, Rees D, Perrin P, Harding RM, Clegg JB, et al. Molecular Analysis of the $\beta$-Globin Gene Cluster in the Niokholo Mandenka Population Reveals a Recent Origin of the $\beta S$ Senegal Mutation. Am J Hum Genet. 2002;70: 207-223. doi:10.1086/338304

3. Jarvis JP, Scheinfeldt LB, Soi S, Lambert C, Omberg L, Ferwerda B, et al. Patterns of Ancestry, Signatures of Natural Selection, and Genetic Association with Stature in Western African Pygmies. PLOS Genet. 2012;8: e1002641. doi:10.1371/journal.pgen.1002641

4. Lachance J, Vernot B, Elbers CC, Ferwerda B, Froment A, Bodo J-M, et al. Evolutionary History and Adaptation from High-Coverage Whole-Genome Sequences of Diverse African Hunter-Gatherers. Cell. 2012;150: 457-469. doi:10.1016/j.cell.2012.07.009

5. Sabeti PC, Varilly P, Fry B, Lohmueller J, Hostetter E, Cotsapas C, et al. Genome-wide detection and characterization of positive selection in human populations. Nature. 2007;449: 913-918. doi:10.1038/nature06250

6. Sabeti PC, Schaffner SF, Fry B, Lohmueller J, Varilly P, Shamovsky O, et al. Positive Natural Selection in the Human Lineage. Science. 2006;312: 1614-1620. doi:10.1126/science.1124309

7. Kelley JL, Swanson WJ. Positive Selection in the Human Genome: From Genome Scans to Biological Significance. Annu Rev Genomics Hum Genet. 2008;9: 143-160. doi:10.1146/annurev.genom.9.081307.164411

8. Fu W, Akey JM. Selection and Adaptation in the Human Genome. Annu Rev Genomics Hum Genet. 2013;14: 467-489. doi:10.1146/annurev-genom-091212-153509

9. Hejase HA, Dukler N, Siepel A. From Summary Statistics to Gene Trees: Methods for Inferring Positive Selection. Trends Genet. 2020;36: 243-258. doi:10.1016/j.tig.2019.12.008

10. Tajima F. Statistical Method for Testing the Neutral Mutation Hypothesis by DNA Polymorphism. Genetics. 1989;123: 585-595.

11. Peter BM, Huerta-Sanchez E, Nielsen R. Distinguishing between Selective Sweeps from Standing Variation and from a De Novo Mutation. PLOS Genet. 2012;8: e1003011. doi:10.1371/journal.pgen.1003011

12. Kern AD, Schrider DR. diploS/HIC: An Updated Approach to Classifying Selective Sweeps. G3 Genes Genomes Genet. 2018;8: 1959-1970. doi:10.1534/g3.118.200262

13. Schrider DR, Kern AD. S/HIC: Robust Identification of Soft and Hard Sweeps Using Machine Learning. PLOS Genet. 2016;12: e1005928. doi:10.1371/journal.pgen.1005928

14. Flagel L, Brandvain Y, Schrider DR. The Unreasonable Effectiveness of Convolutional Neural Networks in Population Genetic Inference. Kim Y, editor. Mol Biol Evol. 2019;36: 
220-238. doi:10.1093/molbev/msy224

15. Torada L, Lorenzon L, Beddis A, Isildak U, Pattini L, Mathieson S, et al. ImaGene: a convolutional neural network to quantify natural selection from genomic data. BMC Bioinformatics. 2019;20: 337. doi:10.1186/s12859-019-2927-x

16. Griffiths RC, Marjoram P. Ancestral inference from samples of DNA sequences with recombination. J Comput Biol J Comput Mol Cell Biol. 1996;3: 479-502. doi:10.1089/cmb.1996.3.479

17. Hudson RR. Gene genealogies and the coalescent process. Oxf Surv Evol Biol. 1990;7: 144.

18. Wiuf C, Hein J. Recombination as a Point Process along Sequences. Theor Popul Biol. 1999;55: 248-259. doi:10.1006/tpbi.1998.1403

19. Hein J. A heuristic method to reconstruct the history of sequences subject to recombination. J Mol Evol. 1993;36: 396-405. doi:10.1007/BF00182187

20. Song YS, Hein J. Constructing Minimal Ancestral Recombination Graphs. J Comput Biol. 2005;12: 147-169. doi:10.1089/cmb.2005.12.147

21. Minichiello MJ, Durbin R. Mapping Trait Loci by Use of Inferred Ancestral Recombination Graphs. Am J Hum Genet. 2006;79: 910-922. doi:10.1086/508901

22. Kuhner MK. LAMARC 2.0: maximum likelihood and Bayesian estimation of population parameters. Bioinformatics. 2006;22: 768-770. doi:10.1093/bioinformatics/btk051

23. O'Fallon BD. ACG: rapid inference of population history from recombining nucleotide sequences. BMC Bioinformatics. 2013;14: 40. doi:10.1186/1471-2105-14-40

24. Rasmussen MD, Hubisz MJ, Gronau I, Siepel A. Genome-Wide Inference of Ancestral Recombination Graphs. PLoS Genet. 2014;10: e1004342. doi:10.1371/journal.pgen.1004342

25. Speidel L, Forest M, Shi S, Myers SR. A method for genome-wide genealogy estimation for thousands of samples. Nat Genet. 2019;51: 1321-1329. doi:10.1038/s41588-019-0484$\mathrm{x}$

26. Kelleher J, Wong Y, Wohns AW, Fadil C, Albers PK, McVean G. Inferring whole-genome histories in large population datasets. Nat Genet. 2019;51: 1330-1338. doi:10.1038/s41588-019-0483-y

27. Arenas $\mathrm{M}$. The importance and application of the ancestral recombination graph. Front Genet. 2013;4. doi:10.3389/fgene.2013.00206

28. LeCun Y, Bengio Y, Hinton G. Deep learning. Nature. 2015;521: 436-444. doi:10.1038/nature14539

29. Hochreiter S, Schmidhuber J. Long Short-Term Memory. Neural Comput. 1997;9: 17351780. doi:10.1162/neco.1997.9.8.1735

30. Maas AL, Daly RE, Pham PT, Huang D, Ng AY, Potts C. Learning Word Vectors for Sentiment Analysis. Proceedings of the 49th Annual Meeting of the Association for Computational Linguistics: Human Language Technologies. Portland, Oregon, USA: Association for Computational Linguistics; 2011. pp. 142-150. Available: https://www.aclweb.org/anthology/P11-1015

31. Hejase HA, Salman-Minkov A, Campagna L, Hubisz MJ, Lovette IJ, Gronau I, et al. Genomic islands of differentiation in a rapid avian radiation have been driven by recent selective sweeps. Proc Natl Acad Sci. 2020;117: 30554-30565. doi:10.1073/pnas.2015987117

32. Garud NR, Messer PW, Buzbas EO, Petrov DA. Recent Selective Sweeps in North American Drosophila melanogaster Show Signatures of Soft Sweeps. PLoS Genet. 2015;11: e1005004. doi:10.1371/journal.pgen.1005004

33. Voight BF, Kudaravalli S, Wen X, Pritchard JK. A Map of Recent Positive Selection in the Human Genome. Hurst L, editor. PLoS Biol. 2006;4: e72.

doi:10.1371/journal.pbio.0040072 
34. Tennessen JA, Bigham AW, O'Connor TD, Fu W, Kenny EE, Gravel S, et al. Evolution and Functional Impact of Rare Coding Variation from Deep Sequencing of Human Exomes. Science. 2012;337: 64-69. doi:10.1126/science.1219240

35. Stern AJ, Wilton PR, Nielsen R. An approximate full-likelihood method for inferring selection and allele frequency trajectories from DNA sequence data. Hernandez RD, editor. PLOS Genet. 2019;15: e1008384. doi:10.1371/journal.pgen.1008384

36. Bersaglieri T, Sabeti PC, Patterson N, Vanderploeg T, Schaffner SF, Drake JA, et al. Genetic Signatures of Strong Recent Positive Selection at the Lactase Gene. Am J Hum Genet. 2004;74: 1111-1120. doi:10.1086/421051

37. Han J, Kraft P, Nan H, Guo Q, Chen C, Qureshi A, et al. A Genome-Wide Association Study Identifies Novel Alleles Associated with Hair Color and Skin Pigmentation. PLOS Genet. 2008;4: e1000074. doi:10.1371/journal.pgen.1000074

38. Sturm RA, Duffy DL, Zhao ZZ, Leite FPN, Stark MS, Hayward NK, et al. A Single SNP in an Evolutionary Conserved Region within Intron 86 of the HERC2 Gene Determines Human Blue-Brown Eye Color. Am J Hum Genet. 2008;82: 424-431. doi:10.1016/j.ajhg.2007.11.005

39. Sulem P, Gudbjartsson DF, Stacey SN, Helgason A, Rafnar T, Magnusson KP, et al. Genetic determinants of hair, eye and skin pigmentation in Europeans. Nat Genet. 2007;39: 1443-1452. doi:10.1038/ng.2007.13

40. Kenny EE, Timpson NJ, Sikora M, Yee M-C, Moreno-Estrada A, Eng C, et al. Melanesian blond hair is caused by an amino acid change in TYRP1. Science. 2012;336: 554. doi:10.1126/science.1217849

41. Liu F, Wollstein A, Hysi PG, Ankra-Badu GA, Spector TD, Park D, et al. Digital Quantification of Human Eye Color Highlights Genetic Association of Three New Loci. PLOS Genet. 2010;6: e1000934. doi:10.1371/journal.pgen.1000934

42. Yoshiura K, Kinoshita A, Ishida T, Ninokata A, Ishikawa T, Kaname T, et al. A SNP in the ABCC11 gene is the determinant of human earwax type. Nat Genet. 2006;38: 324-330. doi:10.1038/ng1733

43. Mathieson S, Mathieson I. FADS1 and the Timing of Human Adaptation to Agriculture. Mol Biol Evol. 2018;35: 2957-2970. doi:10.1093/molbev/msy180

44. Mathieson I. Estimating time-varying selection coefficients from time series data of allele frequencies. bioRxiv. 2020; 2020.11.17.387761. doi:10.1101/2020.11.17.387761

45. Wilde S, Timpson A, Kirsanow K, Kaiser E, Kayser M, Unterländer M, et al. Direct evidence for positive selection of skin, hair, and eye pigmentation in Europeans during the last 5,000 y. Proc Natl Acad Sci. 2014;111: 4832-4837. doi:10.1073/pnas.1316513111

46. Marcus $\mathrm{JH}$, Novembre J. Visualizing the geography of genetic variants. Bioinformatics. 2017;33: 594-595. doi:10.1093/bioinformatics/btw643

47. Turbek SP, Browne M, Giacomo ASD, Kopuchian C, Hochachka WM, Estalles C, et al. Rapid speciation via the evolution of pre-mating isolation in the Iberá Seedeater. Science. 2021;371. doi:10.1126/science.abc0256

48. Campagna L, Repenning M, Silveira LF, Fontana CS, Tubaro PL, Lovette IJ. Repeated divergent selection on pigmentation genes in a rapid finch radiation. Sci Adv. 2017;3: e1602404. doi:10.1126/sciadv.1602404

49. Harding RM, Healy E, Ray AJ, Ellis NS, Flanagan N, Todd C, et al. Evidence for Variable Selective Pressures at MC1R. Am J Hum Genet. 2000;66: 1351-1361. doi:10.1086/302863

50. Ohashi J, Naka I, Tsuchiya N. The Impact of Natural Selection on an ABCC11 SNP Determining Earwax Type. Mol Biol Evol. 2011;28: 849-857. doi:10.1093/molbev/msq264

51. Stern AJ, Speidel L, Zaitlen NA, Nielsen R. Disentangling selection on genetically correlated polygenic traits via whole-genome genealogies. Am J Hum Genet. 2021;108: 219-239. doi:10.1016/j.ajhg.2020.12.005 
52. Kern AD, Schrider DR. Discoal: flexible coalescent simulations with selection. Bioinformatics. 2016;32: 3839-3841. doi:10.1093/bioinformatics/btw556

53. Gal Y, Ghahramani Z. Dropout as a Bayesian Approximation: Representing Model Uncertainty in Deep Learning. International Conference on Machine Learning. PMLR; 2016. pp. 1050-1059. Available: http://proceedings.mlr.press/v48/gal16.html

54. Nachman MW, Crowell SL. Estimate of the mutation rate per nucleotide in humans. Genetics. 2000;156: 297-304.

55. Scally A, Durbin R. Revising the human mutation rate: implications for understanding human evolution. Nat Rev Genet. 2012;13: 745-753. doi:10.1038/nrg3295

56. Schrider DR, Kern AD. Supervised Machine Learning for Population Genetics: A New Paradigm. Trends Genet. 2018;34: 301-312. doi:10.1016/j.tig.2017.12.005

57. Nei M, Li WH. Mathematical model for studying genetic variation in terms of restriction endonucleases. Proc Natl Acad Sci U S A. 1979;76: 5269-5273.

58. Watterson GA. On the number of segregating sites in genetical models without recombination. Theor Popul Biol. 1975;7: 256-276. doi:10.1016/0040-5809(75)90020-9

59. Fay JC, Wu C-I. Hitchhiking Under Positive Darwinian Selection. Genetics. 2000;155: 1405-1413.

60. Messer PW, Petrov DA. Population genomics of rapid adaptation by soft selective sweeps. Trends Ecol Evol. 2013;28: 659-669. doi:10.1016/j.tree.2013.08.003

61. Kelly JK. A Test of Neutrality Based on Interlocus Associations. Genetics. 1997;146: 11971206.

62. Kim Y, Nielsen R. Linkage Disequilibrium as a Signature of Selective Sweeps. Genetics. 2004;167: 1513-1524. doi:10.1534/genetics.103.025387

63. Auton A, Abecasis GR, Altshuler DM, Durbin RM, Abecasis GR, Bentley DR, et al. A global reference for human genetic variation. Nature. 2015;526: 68-74. doi:10.1038/nature15393

64. Campagna L, Gronau I, Silveira LF, Siepel A, Lovette IJ. Distinguishing noise from signal in patterns of genomic divergence in a highly polymorphic avian radiation. Mol Ecol. 2015;24: 4238-4251. doi:10.1111/mec.13314

65. Eriksson N, Macpherson JM, Tung JY, Hon LS, Naughton B, Saxonov S, et al. WebBased, Participant-Driven Studies Yield Novel Genetic Associations for Common Traits. PLOS Genet. 2010;6: e1000993. doi:10.1371/journal.pgen.1000993

66. Lyssenko V, Lupi R, Marchetti P, Guerra SD, Orho-Melander M, Almgren P, et al. Mechanisms by which common variants in the TCF7L2 gene increase risk of type 2 diabetes. J Clin Invest. 2007;117: 2155-2163. doi:10.1172/JCI30706

67. Spellicy CJ, Harding MJ, Hamon SC, Mahoney JJ, Reyes JA, Kosten TR, et al. A variant in ANKK1 modulates acute subjective effects of cocaine: a preliminary study. Genes Brain Behav. 2014;13: 559-564. doi:10.1111/gbb.12121

68. Frayling TM, Timpson NJ, Weedon MN, Zeggini E, Freathy RM, Lindgren CM, et al. A common variant in the FTO gene is associated with body mass index and predisposes to childhood and adult obesity. Science. 2007;316: 889-894. doi:10.1126/science.1141634 


\section{Figures}

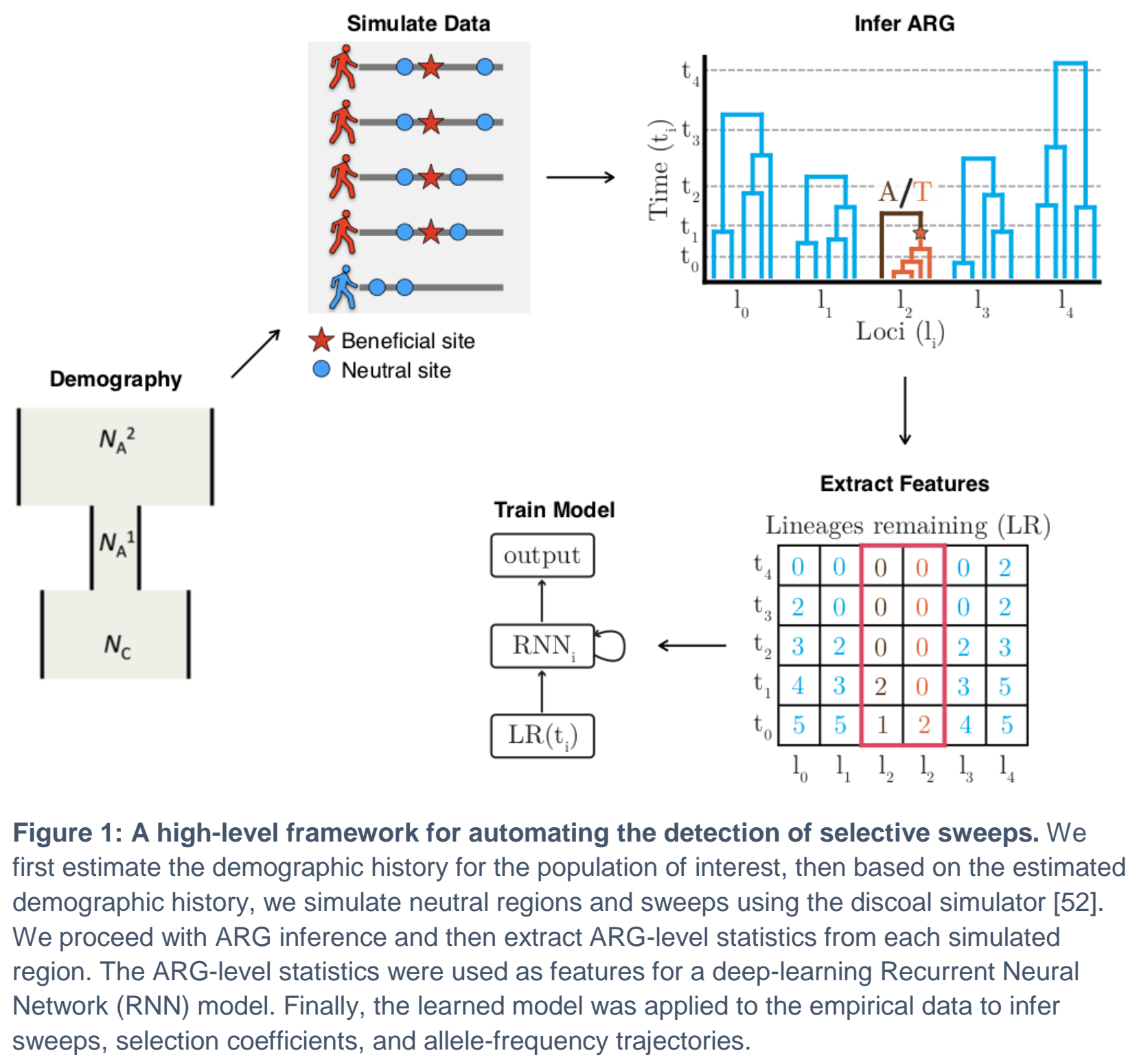


bioRxiv preprint doi: https://doi.org/10.1101/2021.06.22.449427: this version posted June 23 2021. The copyright holder for this preprint (which was not certified by peer review) is the author/funder, who has granted bioRxiv a license to display the preprint in perpetuity. It is made available under aCC-BY-ND 4.0 International license.
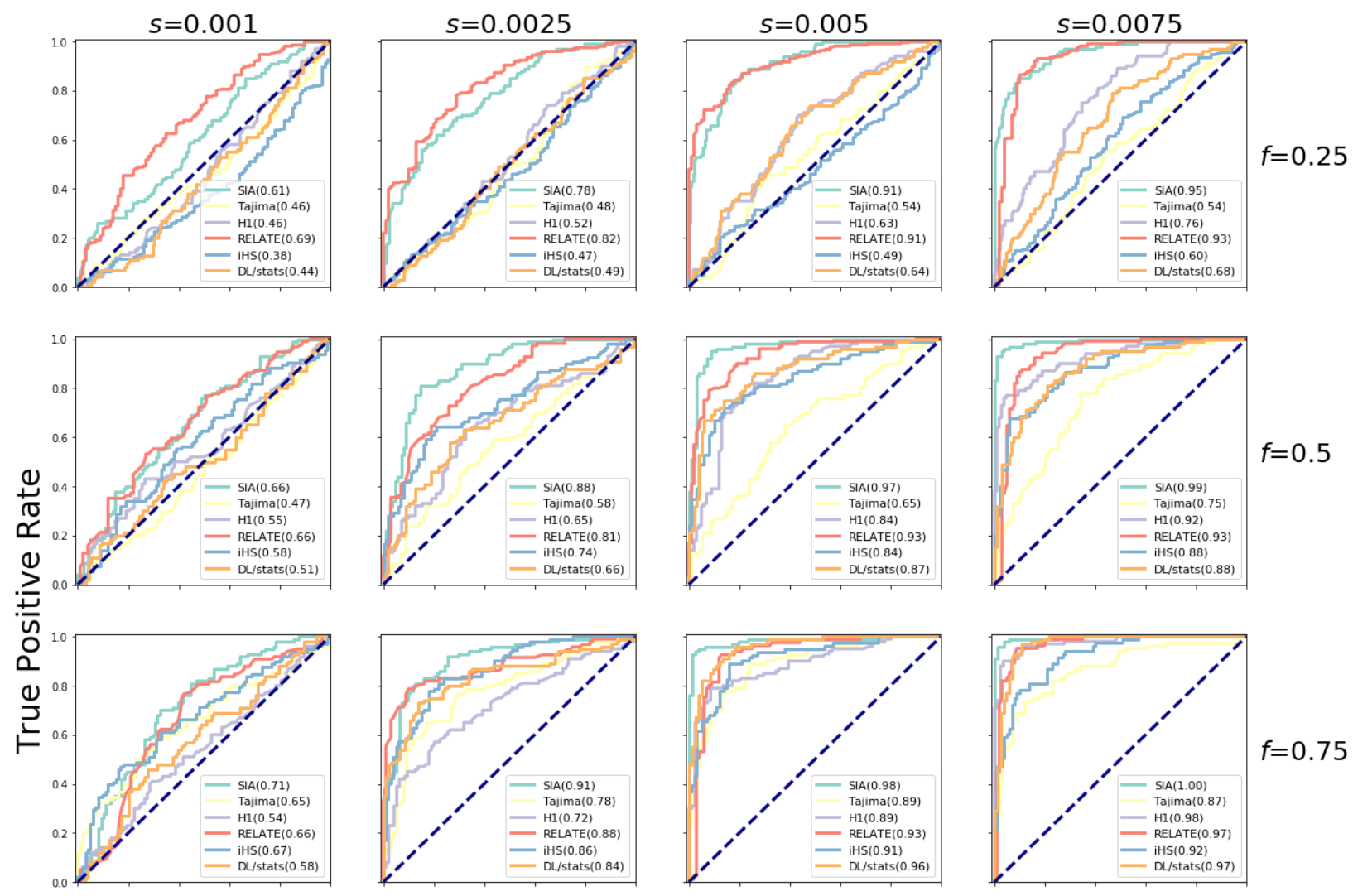

$f=0.75$
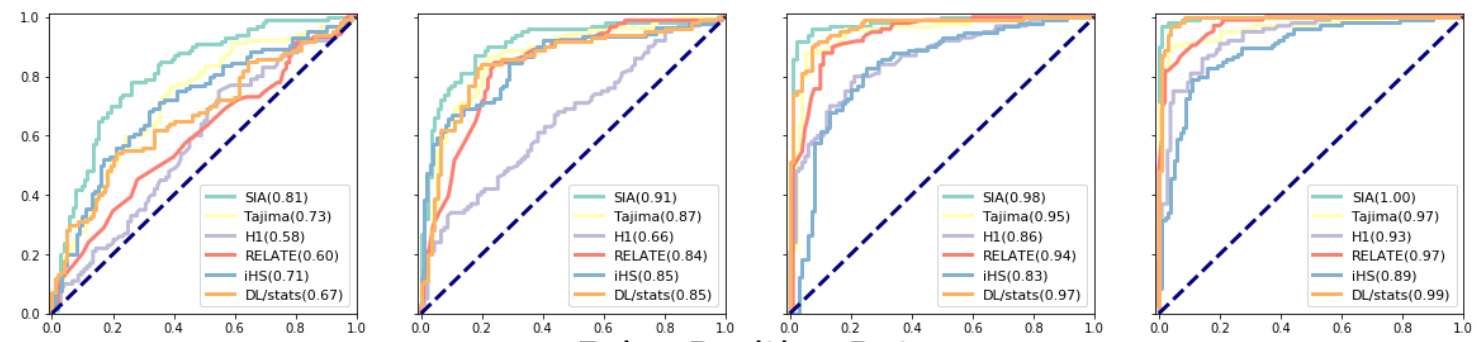

$f=0.9$

674

675

676

677

678

679

680

681

682

683

684

685

Figure 2: Classification performance of SIA and other methods on simulated data.

Sequence data were simulated under a variety of selection regimes ( $s$, shown horizontally) and derived allele frequencies (DAFs) for the beneficial mutation under selection ( $f$, shown vertically) (see Methods for more details). The prediction task distinguished neutral regions and sweeps.

The methods were tested on a set of 200 regions per panel (100 per class), and the receiver operating characteristic (ROC) curve records the true positive rate (TPR) as a function of the false positive rate (FPR). The curve is obtained by varying the prediction threshold from 0 to 1 and recording for each threshold the number of regions correctly assigned (TPS) or misassigned (FPs) as positives (with prediction probability above the threshold). The performance of each method was evaluated based on the area under its ROC curve, or AUROC. We report each method's AUROC as an average across 200 replicate datasets for each model condition. Note that inferred genealogies were used as input to SIA. 
A

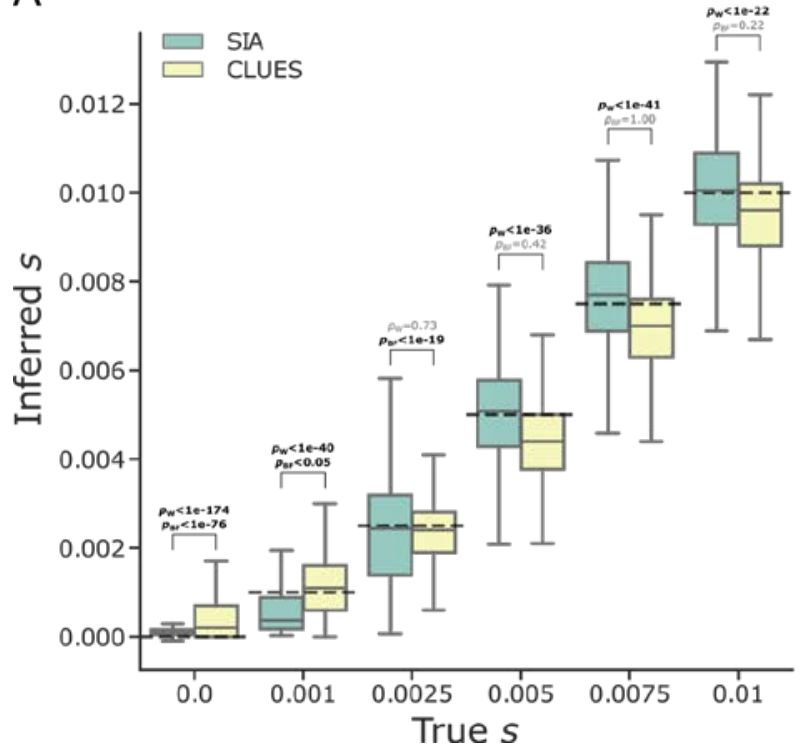

B

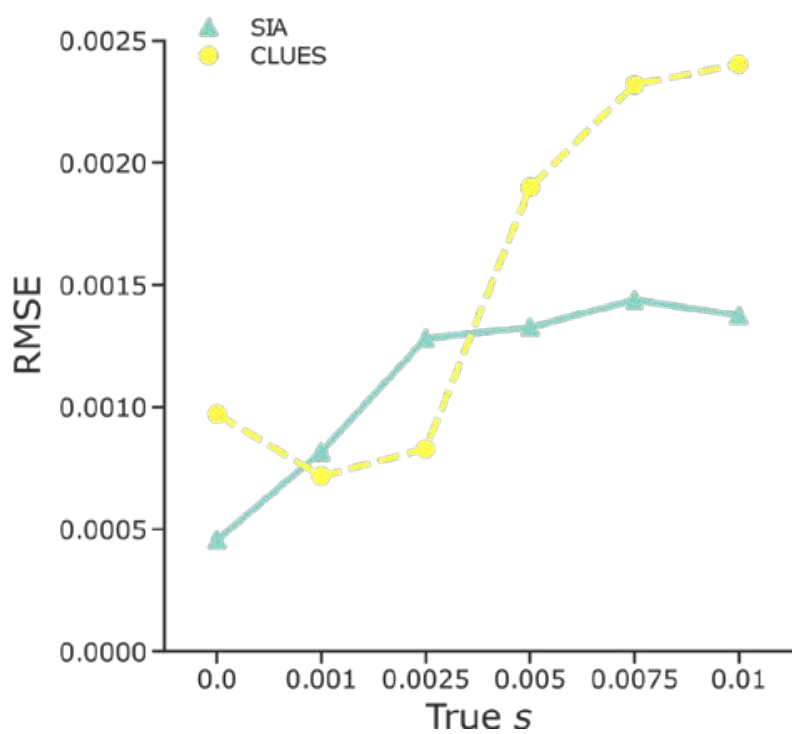

686

687

688

689

690

691

692

693

694

695

696

697

Figure 3: Predictions of selection coefficients for simulated regions using SIA and CLUES based on true genealogies. (A) The distribution of inferred selection coefficients for each method under each model condition are reported using a box plot. The box plot for each method reports these five statistics (from bottom to top): minimum, first quartile, median, third quartile, and maximum. The $y$-axis shows the inferred selection coefficient while the $x$-axis shows the true selection coefficient. The dashed-black line indicates the true selection coefficient for each model condition. The simulations are based on the CEU demographic model and true genealogies were used as input to both methods. Each model condition (i.e. box plot) represents a set of 400 independent simulations. The mean ranks and variances of the distributions of inferred $s$ were compared using the Wilcoxon signed-rank test $\left(p_{\mathrm{W}}\right)$ and the Brown-Forsythe test $\left(p_{\mathrm{BF}}\right)$, respectively. (B) The root mean square error (RMSE) for each method under each model condition evaluated on 400 independent simulations. 
A

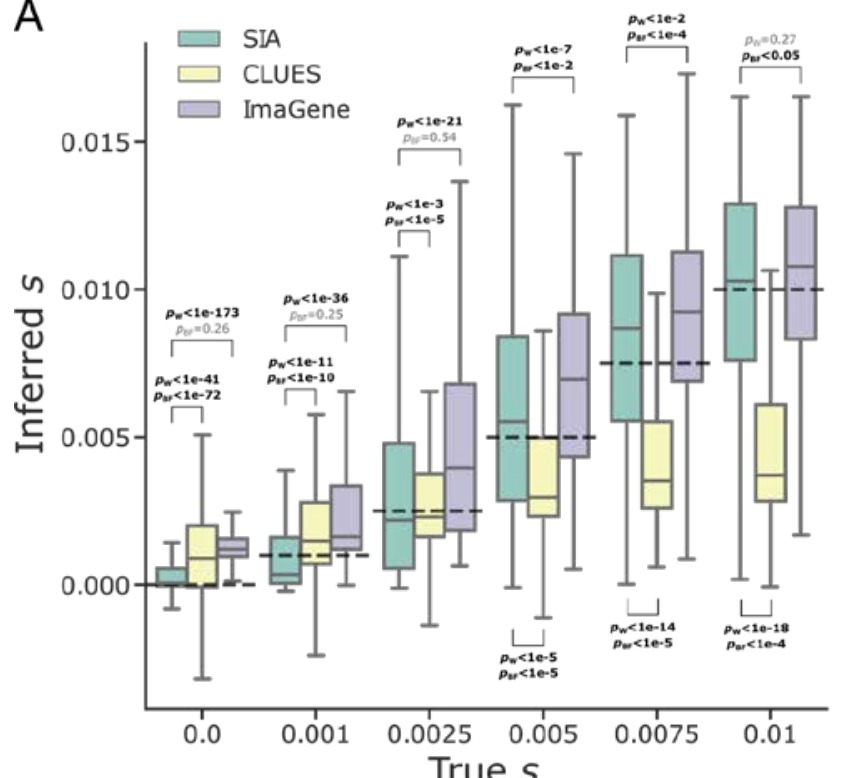

B

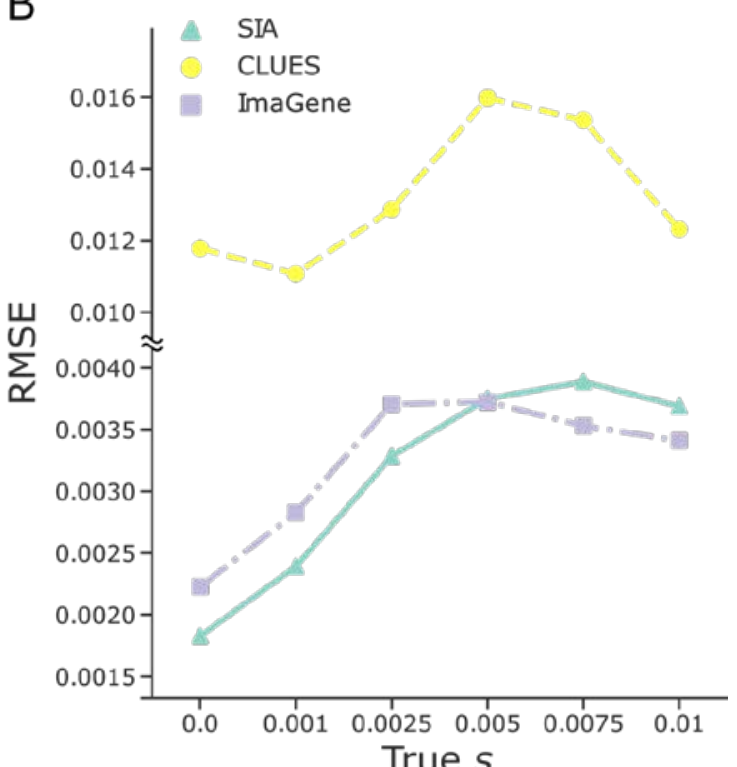

Figure 4: Predictions of selection coefficient on simulated regions using SIA and CLUES based on inferred genealogies, and ImaGene. (A) The distribution of inferred selection coefficients and (B) root mean square error (RMSE) for each method under each model condition. The simulations are based on the CEU demographic model where inferred genealogies were used as input to SIA and CLUES, whereas sequence alignments were used as input to ImaGene. Figure layout and description are otherwise similar to Figure 3. 
LCT

rs4988235

Derived frequency $=0.74$

Prob $=0.99$

$s=[0.01019,0.01056]$

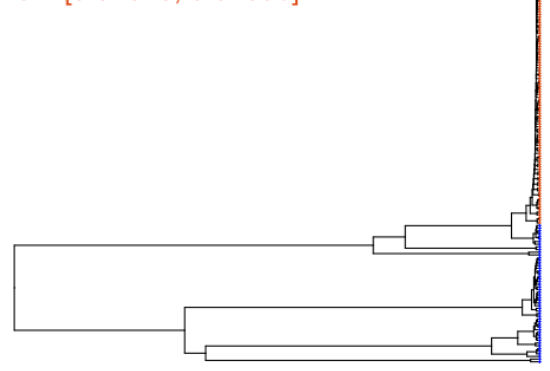

\section{MC1R}

rs1805007

Derived frequency $=0.12$

Prob $=0.949$

$s=[0.00362,0.00384]$

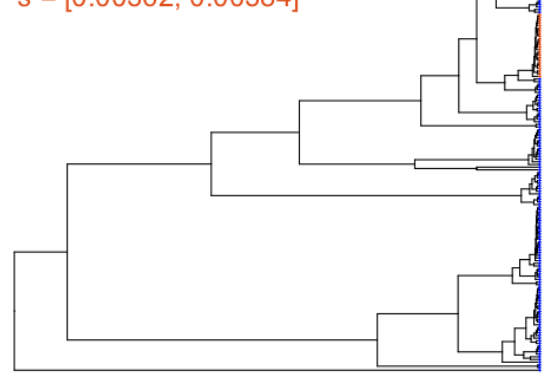

ASIP

rs619865

Derived frequency $=0.12$

Prob $=0.777$

$s=[0.00172,0.00197]$

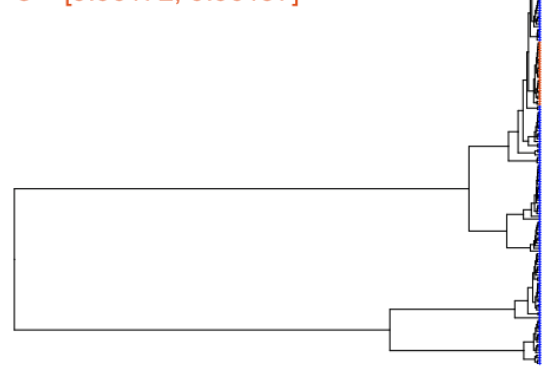

OCA2

rs12913832

Derived frequency $=0.77$

Prob $=0.75$

$s=[0.00539,0.00575]$

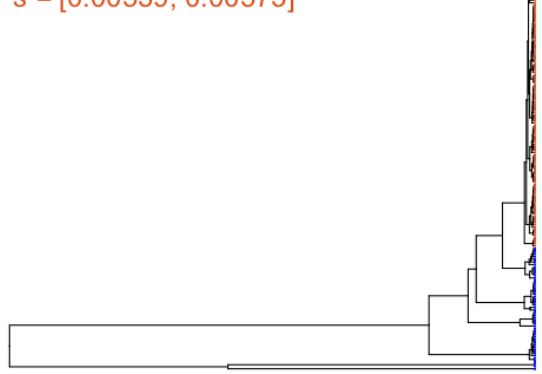

\section{$\mathrm{ABCC} 11$}

rs17822931

Derived frequency $=0.13$

Prob $=0.620$

$s=[0.00034,0.00036]$

TYR

rs1393350

Derived frequency $=0.24$

Prob $=0.616$

$s=[0.00085,0.00135]$

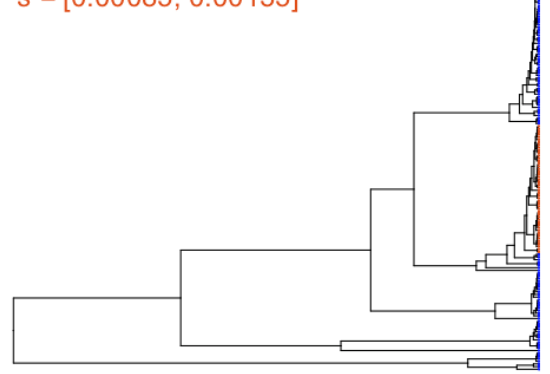

704 Figure 5: Local genealogies at six loci inferred to be under positive selection in the 1000

705 Genomes CEU population. Gene name, RefSNP number, derived allele frequency, SIA-

706 inferred sweep probability and SIA-inferred selection coefficient range for each locus are

707 indicated at the top of each panel (see Table 1 for more details). Taxa carrying the ancestral

708 and derived alleles are colored in blue and orange, respectively. 

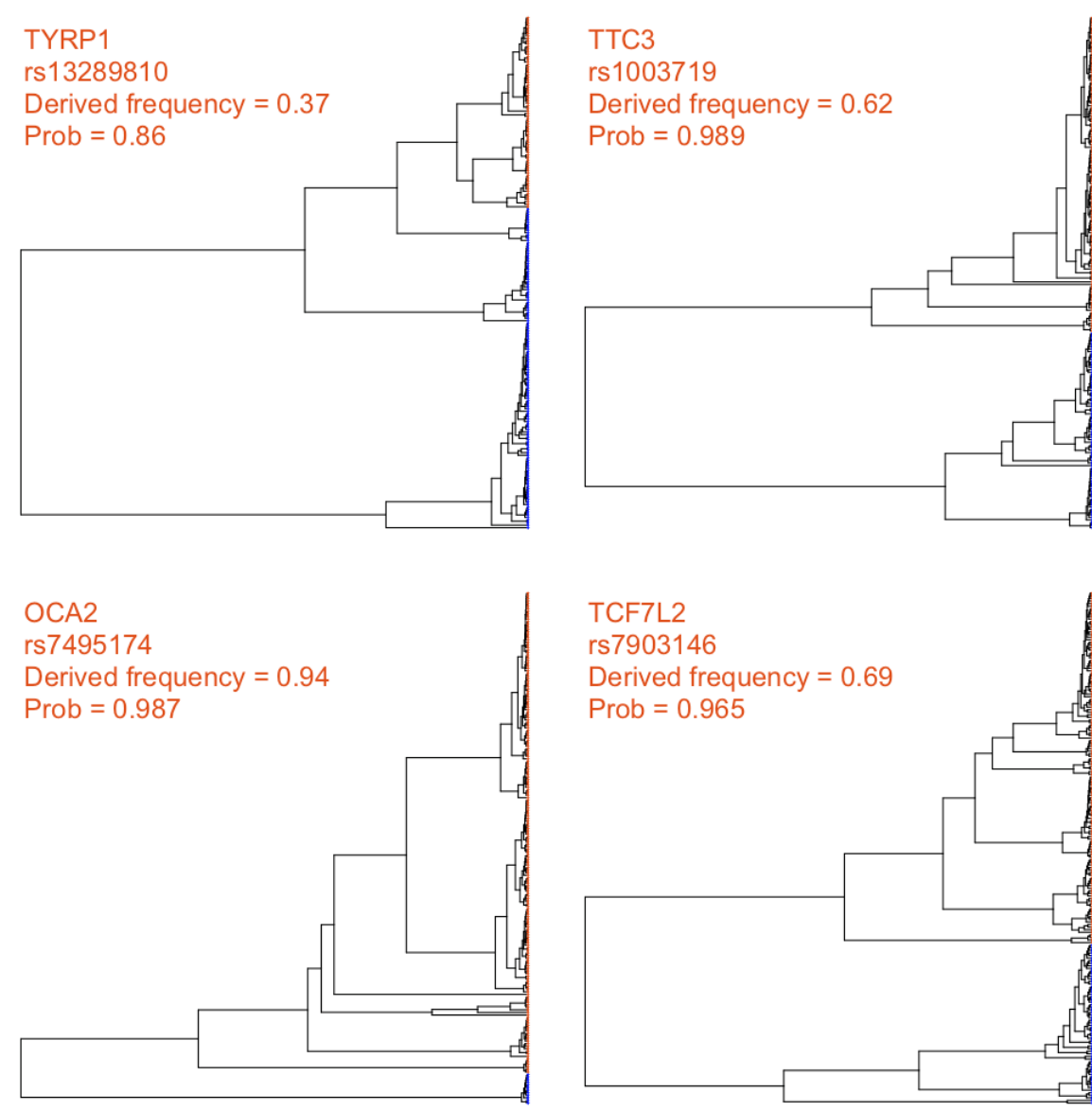

\section{TCF7L2} rs7903146

Derived frequency $=0.69$

Prob $=0.965$
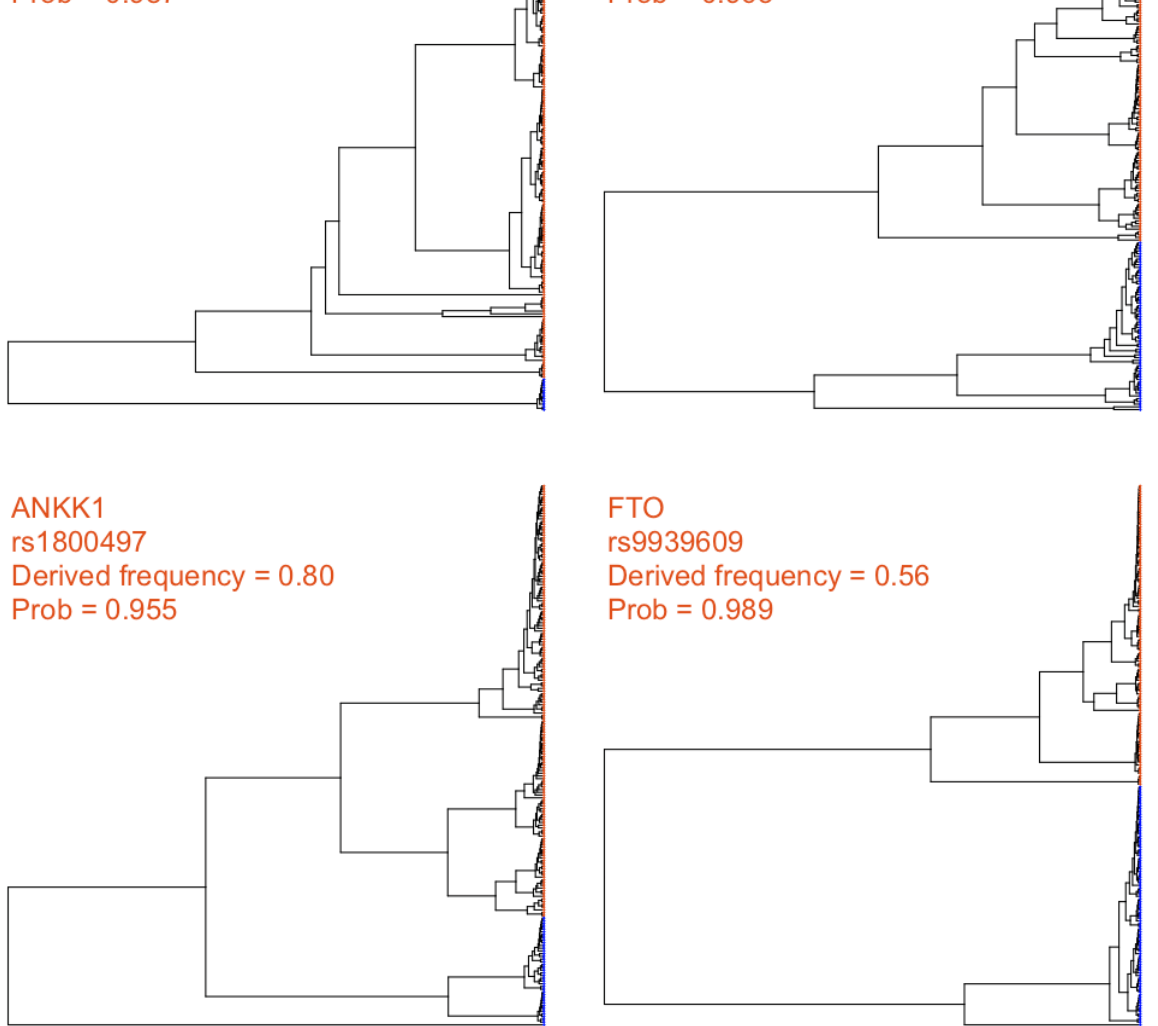

FTO

rs9939609

Derived frequency $=0.56$

Prob $=0.989$

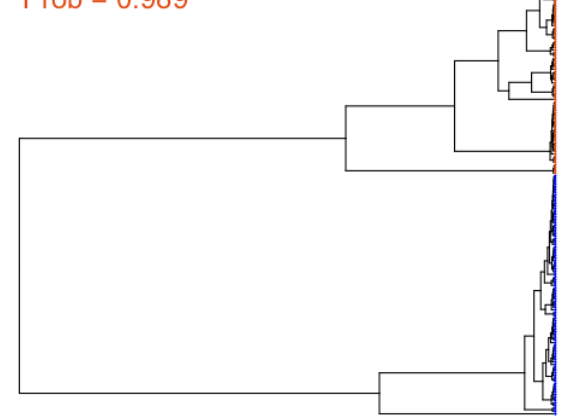

709 Figure 6: Local genealogies at six loci lacking signal of positive selection in the 1000

710 Genomes CEU population. Gene name, RefSNP number, derived allele frequency and

711 probability of neutrality inferred by SIA for each locus are indicated at the top of each panel (see

712 Table 1 for more details). Taxa carrying the ancestral and derived alleles are colored in blue

713 and orange, respectively. 

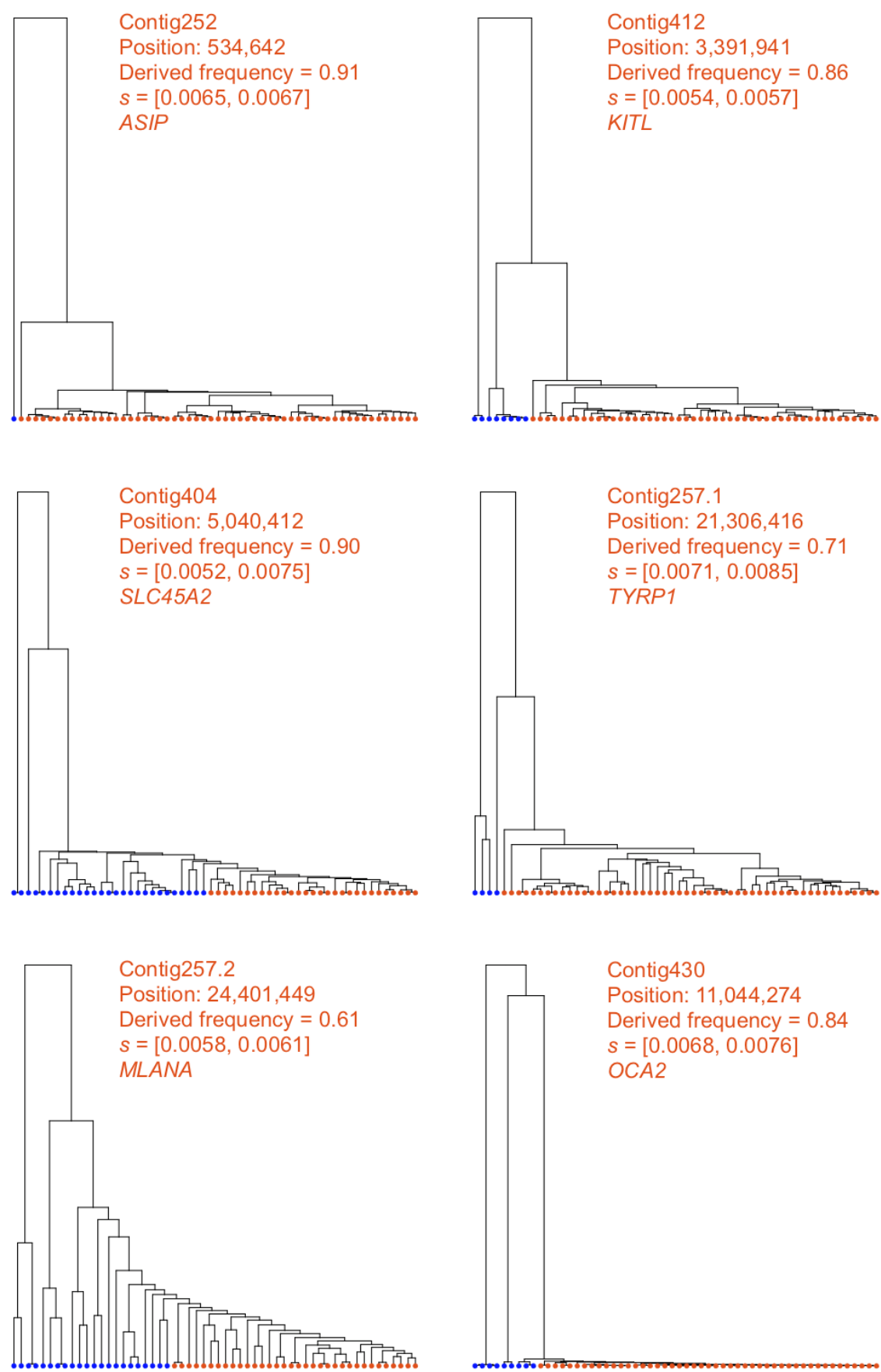

714 Figure 7: Local genealogies at five loci inferred to be under positive selection in S.

715 hypoxantha. Contig name, position of SNP, derived allele frequency, SIA-inferred selection

716 coefficient range, and the pigmentation gene closest to the locus in question are indicated at the 717 top of each panel. Haploid genomes carrying the ancestral and derived alleles are colored in 718 blue and orange, respectively. 


\section{Tables}

Table 1: List of genomic loci of interest along with their derived allele frequencies (DAF), sweep probabilities, and selection coefficients inferred by SIA in the 1000 Genomes CEU population.

\begin{tabular}{|l|l|l|l|l|l|l|}
\hline \multicolumn{1}{|c|}{ Gene } & \multicolumn{1}{|c|}{ SNP ID } & Chr & Position* & DAF & P(sweep) & $\begin{array}{c}\text { Selection } \\
\text { coefficient (95\% Cl) }\end{array}$ \\
\hline LCT [36] & rs4988235 & 2 & 136608646 & 0.74 & 0.999 & {$[0.01019,0.01056]$} \\
\hline OCA2 [37,38] & rs12913832 & 15 & 28365618 & 0.77 & 0.750 & {$[0.00539,0.00575]$} \\
\hline MC1R [37,39] & rs1805007 & 16 & 89986117 & 0.12 & 0.949 & {$[0.00362,0.00384]$} \\
\hline ABCC11 [42] & rs17822931 & 16 & 48258198 & 0.13 & 0.620 & {$[0.00034,0.00036]$} \\
\hline ASIP [65] & rs619865 & 20 & 33867697 & 0.12 & 0.777 & {$[0.00172,0.00197]$} \\
\hline TYR [39,65] & rs1393350 & 11 & 89011046 & 0.24 & 0.616 & {$[0.00085,0.00135]$} \\
\hline KITLG [39] & rs12821256 & 12 & 89328335 & 0.13 & 0.869 & {$[0.00183,0.002]$} \\
\hline TYRP1 [40] & rs13289810 & 9 & 12396731 & 0.37 & 0.144 & {$[0.00004,0.00006]$} \\
\hline TTC3 [41] & rs1003719 & 21 & 38491095 & 0.62 & 0.011 & {$[0,0]$} \\
\hline OCA2 & rs7495174 & 15 & 28344238 & 0.94 & 0.013 & {$[0,0.00005]$} \\
\hline TCF7L2 [66] & rs7903146 & 10 & 114758349 & 0.69 & 0.035 & {$[0,0]$} \\
\hline ANKK1 [67] & rs1800497 & 11 & 113270828 & 0.80 & 0.045 & {$[0,0]$} \\
\hline FTO [68] & rs9939609 & 16 & 53820527 & 0.56 & 0.011 & {$[0,0]$} \\
\hline
\end{tabular}


Table 2: The top $25 F_{\text {ST }}$ peaks identified in [31] along with the number of partial soft sites in S. hypoxantha identified for each scaffold using SIA. To avoid cases with limited power, we focused on sites with segregating frequency $\geq 0.5$, SIA-inferred $s>0.0025$, and SIA-inferred sweep probability $\geq 0.99$.

730

\begin{tabular}{|c|c|c|c|c|}
\hline Scaffold & Start position (Mb) & End position (Mb) & Length (kb) & \# of partial soft sites* \\
\hline 59 & 5.74 & 5.86 & 120 & 11 \\
\hline 118 & 7.16 & 7.22 & 60 & 5 \\
\hline 252 & 0.40 & 0.54 & 140 & 3 \\
\hline 257.1 & 21.24 & 21.78 & 540 & 26 \\
\hline 257.2 & 24.40 & 24.84 & 440 & 43 \\
\hline 257.3 & 28.66 & 28.96 & 300 & 10 \\
\hline 257.4 & 31.30 & 31.38 & 80 & 8 \\
\hline 257.5 & 5.78 & 6.20 & 420 & $25(1)$ \\
\hline 263 & 0.00 & 0.58 & 580 & 31 \\
\hline 308 & 0.04 & 0.20 & 160 & 0 \\
\hline 404.1 & 5.04 & 5.84 & 800 & $115(7)$ \\
\hline 404.2 & 10.76 & 10.96 & 200 & 30 \\
\hline 412 & 3.38 & 3.62 & 240 & 15 \\
\hline 430 & 10.98 & 11.10 & 120 & 24 \\
\hline 567 & 2.50 & 2.80 & 300 & 0 \\
\hline 637.1 & 6.00 & 6.32 & 320 & 2 \\
\hline 637.2 & 6.84 & 6.92 & 80 & 4 \\
\hline 762 & 1.65 & 1.73 & 80 & 30 \\
\hline 766 & 1.98 & 2.10 & 120 & 1 \\
\hline 791 & 9.90 & 9.98 & 80 & 15 \\
\hline 1717 & 0.92 & 0.98 & 60 & 7 \\
\hline 3622 & 0.96 & 1.36 & 400 & 8 \\
\hline 1635 & 3.71 & 3.75 & 40 & 4 \\
\hline 1954 & 2.8 & 2.9 & 100 & 17 \\
\hline 579 & 0.1 & 0.16 & 60 & 0 \\
\hline
\end{tabular}

731 Note: *The number of sweep sites in coding regions is shown in parenthesis. 